\title{
Monitoring Microbe-Induced Physical Property Changes Using High-Frequency Acoustic Waveform Data: Toward the Development of a Microbial Megascope
}

by

Kenneth Hurst Williams

B.A. (University of California, Berkeley), 1993

A thesis submitted in partial satisfaction of the requirements for the

degree of

Master of Science

in

Geophysics

in the

GRADUATE DIVISION

of the

UNIVERSITY OF CALIFORNIA, BERKELEY

\author{
Committee in charge: \\ Professor Lane R. Johnson, Chair \\ Professor Jillian F. Banfield \\ Professor Mary K. Firestone
}

Spring 2002

This work was supported by the Director, Office of Science, Office of Biological and Environmental Research, Environmental Sciences Division, Natural and Accelerated Bioremediation Research Program, of the U.S. Department of Energy under Contract No. DE-AC03-765F0098. 


\title{
Abstract
}

\section{Monitoring Microbe-Induced Physical Property Changes Using}

\author{
High-Frequency Acoustic Waveform Data: Toward the
}

\author{
Development of a Microbial Megascope \\ by
}

\author{
Kenneth Hurst Williams \\ Master's of Science in Geophysics \\ University of California, Berkeley \\ Professor Lane R. Johnson, Chair
}

A laboratory investigation was undertaken to determine the effect of microbe generated gas bubbles in controlled, saturated sediment columns utilizing a novel technique involving acoustic wave propagation. Specifically, the effect of denitrifying bacteria on saturated flow conditions was evaluated in light of the stimulated production of $\mathrm{N}_{2}$ gas and the resulting plugging of the pore throats. The propagation of high frequency acoustic waves through the sediment columns was used to locate those regions in the column where gas accumulation occurred. Over a period of six weeks, regions of gas accumulation resulted in the attenuation of acoustic wave energies with the decreases in amplitude typically greater 
than one order of magnitude. The temporal production of $\mathrm{N}_{2}$ gas was evaluated quantitatively using the stable isotope ${ }^{15} \mathrm{~N}$ in the form of added $\mathrm{Na}^{15} \mathrm{NO}_{3}$. This was done to ascertain the origin (biotic or abiotic) of any produced gas with the results showing a dramatic increase in microberespired ${ }^{15} \mathrm{~N}_{2}$. Hydraulic conductivity $\left(\mathrm{K}_{\mathrm{s}}\right)$ measurements made over the experimental period establish the rate and degree of pore throat blocking with the result being a reduction in $\mathrm{K}_{\mathrm{s}}$ by more than 70 percent. The results were compared to a stimulated but non-inoculated control column which showed neither a decrease in acoustic wave amplitudes nor hydraulic conductivity over the same time-period. Final destructive analysis of one column was performed in order to assess the cell density of denitrifying microbes throughout the column. Cell densities were found to be in close agreement with the stoichiometric predictions made prior to initiation of the experiment. Evaluation of the multiple data sets suggests that microbial gas production is both directly detectable using the high frequency acoustic wave approach and capable of significantly altering saturated flow conditions. 


\section{Contents}

List of Figures $\quad$ iii

List of Tables ix

Acknowledgements $\quad \mathrm{x}$

1 Introduction 1

2 Experimental Approach and Methods $\quad 8$

2.1 Introduction 8

2.2 Assimilatory and Dissimilatory Half Reactions 9

$\begin{array}{lll}2.3 & \text { Laboratory Studies } & 10\end{array}$

$\begin{array}{lll}2.3 .1 & \text { Sediment Selection } & 12\end{array}$

2.3.2 Microbial Growth and Inoculation 12

2.3.3 Saturation of the Columns \& Nutrient Addition $\quad 17$

2.3.4 Hydraulic Conductivity Measurements 21

2.3.5 Gas Sampling Measurements 22

2.3.6 Acoustic Wave Measurements 24

2.3.7 Microbial Biomass Estimation - Denitrification Assay 29

$3 \quad$ Experimental Data and Results 31

3.1 Saturated Hydraulic Conductivity Measurements 31

3.2 Atom $\%{ }^{15} \mathrm{~N}$ Gas Sampling 35

3.3 Acoustic Wave Measurements 38

3.3.1 Acoustic Waveform Data 39

3.3.2 Acoustic Wave Amplitude Data 50

3.3.3 Acoustic Wave Attenuation Coefficients 54 
3.3.4 Acoustic Wave Spectra 59

3.4 Microbial Biomass Estimation - Denitrification Assay 63

4 Discussion and Conclusions 66

4.1 Introduction 66

4.2 Assessment of Temporal Column Conditions 67

4.3 Acoustic Estimation of Bubble Development 68

$\begin{array}{lll}4.4 \text { Conclusions } & 77\end{array}$

$\begin{array}{ll}\text { References } & 79\end{array}$

$\begin{array}{ll}\text { Appendix A } & 85\end{array}$ 


\section{List of Figures}

1.1 Illustration showing the idealized development of respired gas bubbles within the pore spaces of saturated sediments. Note the variety of bubble shapes that may exist both within and between pore

1.2 Illustration showing the potential layout of a cross-well geophysical system designed to monitor terrestrial subsurface microbial activity over time. Only a few of the many potential acoustic wave paths are shown. Note that changes in subsurface conditions may arise through a variety of means, not solely gas production. Attenuation of acoustic wave energy through a region of gas production is shown in b) for the conditions depicted in c).

2.1 Photograph showing three of the columns used in the experiment and the general design of each. The features shared by all of the columns are clear Polycarbonate chambers, gas and water tight seals, removable end caps, and adjustable influent and effluent lines.

2.2 Photograph showing the general arrangement of the carbon dioxide flushing system. Gas flowed from the cylinder through a calibrated flow indicator (mounted on the ring stand to the lower left of the column) and upward through the column displacing the less dense ambient pore space air. 
2.3 Photograph showing the assembly used to make measurements of saturated hydraulic conductivity. Note the modified Mariotte bottle mounted on the ring stand above and to the right of the column. The $\Delta \mathrm{h}$ value is determined by measuring the distance from the bubble tube (inside the fluid reservoir) to the outflow (inside the graduated cylinder).

2.4 Photograph showing the assembly used to extract gas samples from within the column. The gas permeation samplers were constructed of silicon tubing (red), inserted through the column wall, and connected to barbed gas chromatograph septa valves (metal/green). Gas diffused from the column through the tubing and was withdrawn by syringe.

2.5 Photograph showing the apparatus designed to make acoustic wave measurements. The sediment column was inserted into a waterfilled tube into which the acoustic transducers were coupled. The transducers (piezoelectric cylinders with black connecting cables) remain fixed while the column was raised/lowered within the tube (measurement locations are visible).

2.6 Photograph showing the apparatus designed to make acoustic wave measurements from the top. The $180^{\circ}$ alignment of the transducers is visible as is their offset from the column wall.

3.1 The results of the saturated hydraulic conductivity $\left(\mathrm{K}_{\mathrm{s}}\right)$ measurements over the course of the experiment. The first indication of gas within the acoustic monitoring column is show for comparison. Note that very little decrease in $\mathrm{K}_{\mathrm{s}}$ occurs before this time but that the values drop rapidly once gas appears, ultimately stabilizing to a final value. The results from the control column are also shown for comparison. 
3.2 Close-up photograph of the acoustic monitoring column after 60 days have elapsed since saturation. The bubble accumulation observed in this column was similar to that observed in the other inoculated columns. Its dimensions were approximately $2 \mathrm{~cm} \times 3 \mathrm{~cm}$ $\mathrm{x} 1 \mathrm{~cm}$ and it possibly represents entrapment and accumulation along a packing discontinuity.

3.3 The results of the isotope ratio mass spectrometer analysis of evolved column ${ }^{15} \mathrm{~N}$. The gas sampled over the first two weeks of the experiment shows no deviation from atmospheric atom $\%{ }^{15} \mathrm{~N}$ (0.3663) but breakthrough of labeled gas appears to transpire once pressure within the column rises enough to enhance diffusion into the samplers. The final results indicate that respired $\mathrm{N}_{2}$ was the only reasonable source of gas within the columns.

3.4 Acoustic waveform data from the microbe inoculated column for the $24 \mathrm{~cm}$ depth location over four periods of time. Note the travel time differences and amplitude decreases as a function of time and gas evolution.

3.5 Acoustic waveform data from the microbe inoculated column for the $16 \mathrm{~cm}$ depth location over four periods of time. Note the travel time differences and amplitude decreases as a function of time and gas evolution.

3.6 Acoustic waveform data from the microbe inoculated column for the $8 \mathrm{~cm}$ depth location over four periods of time. Note the travel time differences and amplitude decreases as a function of time and gas evolution.

3.7 Acoustic waveform data from the microbe inoculated column for the $4 \mathrm{~cm}$ depth location over four periods of time. Note the travel time differences and amplitude decreases as a function of time and gas evolution. 
3.8 Acoustic waveform data from the control column for the $12 \mathrm{~cm}$ depth location over two periods of time. Note the high degree of repeatability of the waveforms. Such repeatability suggests that the effects observed in the inoculated column are exclusively biogenic in origin as the control column received the same saturating medium.

3.9 Acoustic waveform data from the control column for the $10 \mathrm{~cm}$ depth location over two periods of time. Note the high degree of repeatability of the waveforms. Such repeatability suggests that the effects observed in the inoculated column are exclusively biogenic in origin as the control column received the same saturating medium.

3.10 Acoustic waveform data from the control column for the $8 \mathrm{~cm}$ depth location over two periods of time. Note the high degree of repeatability of the waveforms.

3.11 Acoustic waveform data from the control column for the $6 \mathrm{~cm}$ depth location over two periods of time. Note the high degree of repeatability of the waveforms.

3.12 Acoustic waveform data from the inoculated column for the $24 \mathrm{~cm}$ depth location over two periods of time. The voltage scale for the attenuated signal (Day 28) is shown on the right. Note the differences in wave travel time.

3.13 Acoustic waveform data from the inoculated column for the $16 \mathrm{~cm}$ depth location over two periods of time. The voltage scale for the attenuated signal (Day 28) is shown on the right. Note the differences in wave travel time.

3.14 Acoustic waveform data from the inoculated column for the $8 \mathrm{~cm}$ depth location over two periods of time. The voltage scale for the attenuated signal (Day 28) is shown on the right. Note the differences in travel times. 
3.15 Acoustic waveform data from the inoculated column for the $4 \mathrm{~cm}$ depth location over two periods of time. The voltage scale for the attenuated signal (Day 28) is shown on the right. Note the differences in travel times.

3.16 Series of graphs illustrating change in the measured wave amplitudes over the four periods shown in Figures 3.4 to 3.7. The onset of gas at the $8 \mathrm{~cm}$ location is followed by abrupt gas production throughout the column shortly thereafter. Note the lack of attenuation of those waves transmitted through the uppermost portion of the column.

3.17 Graph of changing wave amplitudes over the experiment for both the microbe-inoculated and the control column. Note the lower values both high and low in the control column which are likely due to initially entrapped air during saturation. There is no observed temporal change, however.

3.18 Acoustic waveform data illustrating the difference in wave amplitude and travel time for a wave transmitted through a sediment-filled and a water-filled column. The amplitude from the latter was used to determine the attenuation coefficient of the sediment alone (i.e. no gas).

3.19 Inoculated column waveform spectra for the $16 \mathrm{~cm}$ location over three time periods (Day 28 voltage scale is on the right). Note the selective attenuation of frequencies above $950 \mathrm{kHz}$. These correspond to damping losses associated with very small diameter bubbles or more likely, scattering off of larger diameter bubbles. 60

3.20 Inoculated column waveform spectra for the $8 \mathrm{~cm}$ location over three time periods (Day 28 voltage scale is on the right). Note the selective attenuation of frequencies between 750 and $950 \mathrm{kHz}$ and above $1 \mathrm{MHz}$. These may correspond to both damping losses 
associated with a range of pore-filling bubble sizes and to wave scattering.

3.21 Control column waveform spectra for the $12 \mathrm{~cm}$ location over two time periods. Note the high degree of repeatability in the two spectra over the course of nearly one month.

4.1 Mathematical estimation of the resonance frequencies corresponding to a wide range of bubble sizes (lower image is an expanded view). The parameters used here match those believed to exist within the columns.

4.2 Modeled prediction of wave attenuation coefficients in fine sand for a wide range of frequencies (modified from Tuffin et al., 2000). Note that the conditions modeled here are not an exact analog for those in the columns. Rather the figure is included to illustrate the dramatic increase in attenuation possible when transmitting frequencies closely match the particular resonance frequency of bubbles in the sediment. 


\section{List of Tables}

$3.1 \quad$ Atom $\%{ }^{15} \mathrm{~N}$ Values 35

3.2 Amplitude Data 51

3.3 Attenuation coefficients for the column over time 56

3.4 Attenuation of saturated vs. gassy sediments 57

3.5 MPN of denitrifying bacteria per gram wet weight 65

4.1 Parameters used in resonance frequency calculation 72

A.1 Sediment Parameters 85

A.2 Sediment Column Dimensions 86

A.3 Composition of 100x Medium D Stock Solutions 86

A.4 Proportions of Stock Solutions for 1L 1000x Medium D 88 


\section{Acknowledgements}

My most sincere thanks go to the people who have made this experience possible. Without the help of the ever resourceful and always curious Berkeley faculty, students, and staff, I would have likely abandoned this endeavor some time ago.

My advisor, Lane Johnson, has been of tremendous support in this effort. He never once balked at my request to take what appeared to be innumerable microbiology courses. He always kept the goals in sight and allowed me to bring the project along on my own schedule. I find it hard to imagine another advisor who would have allowed me such control, particularly on such an esoteric project as this one.

As this project grew directly out of a Berkeley class project, I am indebted to those who made ESPM 127 a reality. They include the three faculty members, Mary Firestone, Harvey Doner, and Masoud Ghodrati, without whom the class would have suffered. Their enthusiasm and effort both during the course and in the subsequent semesters have made this undertaking possible. Also to be thanked are Don Herman and Brandy 
Toner, both of whom put in countless hours to help get me started in a field that I had very little prior experience. The University should be proud to have attracted such people and to have offered up a course that truly does make an impact.

I would also like to thank my friends and colleagues at the Lawrence Berkeley National Lab, in particular Ernie Majer, Susan Hubbard, Tom Daley, John Peterson, Jil Geller, Don Lippert, and Phil Rizzo. At some point along the way, each of them has made a difference that has made earning my degree easier than it would have otherwise been. Thanks also go to Walter Denn for his assistance in preparing several figures.

Finally, I am very appreciative of those who have helped review this thesis in its final stages, in particular Lane. It is a much better document for it. Thanks to my thesis committee for hanging in there until the final minutes. I look forward to my future endeavors with Professors Jill Banfield and Mary Firestone and hope that this thesis represents the first step in a much bigger journey. I would also like to thank Professor Gary Sposito for his clever coining of the phrase "Microbial Megascope."

I dedicate this thesis to my parents and grandparents who have willed me toward getting this degree. I'm better for it. I also dedicate this thesis to my girlfriend, Janelle, who has seen me through the past three stressful yet rewarding years. I hope I can give her the same love and support during her journey as she has given me. 


\section{Chapter 1}

\section{Introduction}

A large amount of data have been reported providing evidence that microbe mediated processes can lead to the production of gases in saturated porous media under both field and laboratory conditions [Doner et. al., 1974, 1975b; Doner 1975a; Doner and McClaren 1978; Vogel et. al., 1981; Trudell et. al., 1986; Andrews and Wilson, 1987; Wilson et. al., 1990; Baveye et. al., 1998]. A portion of this work has focussed on understanding the impact of such biogenic gases on subsurface flow properties, such as saturated hydraulic conductivity $\left(\mathrm{K}_{\mathrm{s}}\right)$. The work done to date has evaluated the process in materials ranging from sands to blanket peat [Ronen et. al., 1998; Reynolds et. al., 1992; Beckwith and Baird, 2001]. In particular, efforts have been made to establish a 
connection between microbial gases produced within pore spaces and the obstruction of fluid flow pathways. The resulting decrease in $\mathrm{K}_{\mathrm{s}}$ is often attributed to the blocking of pore throats by accumulated gas bubbles with the available data suggesting that $\mathrm{K}_{\mathrm{S}}$ may be lowered by as much as an order of magnitude [Reynolds et. al., 1992; Beckwith and Baird, 2001]. The implications for fluid flow in the region of the gas-blocked pore throats are such that flow may be either redirected or blocked altogether. The effect is shown schematically in Figure 1.1. Such an effect must therefore be taken into consideration when predictions are made of flow in the saturated subsurface environment.

It has been recognized by individuals working in the area of subsurface contaminant remediation that changes in the pore fluid composition which result in altered flow properties may require the reconsideration of treatment options [Smith et. al., 2001; Wilson et. al., 2002]. Regions in which contaminants are present may be prevented from being accessed and remediated by a particular treatment method through gaseous pore throat blocking and permeability alteration [Chapelle et al., 1987]. Treatment options that enhance the activity of subsurface denitrifying microbes or other microbes capable of producing insoluble gases may result in the production of unmanageable quantities of gas. At present, no characterization technique exists that allows for detailed, high-resolution imaging of those regions in the terrestrial (i.e. non- 


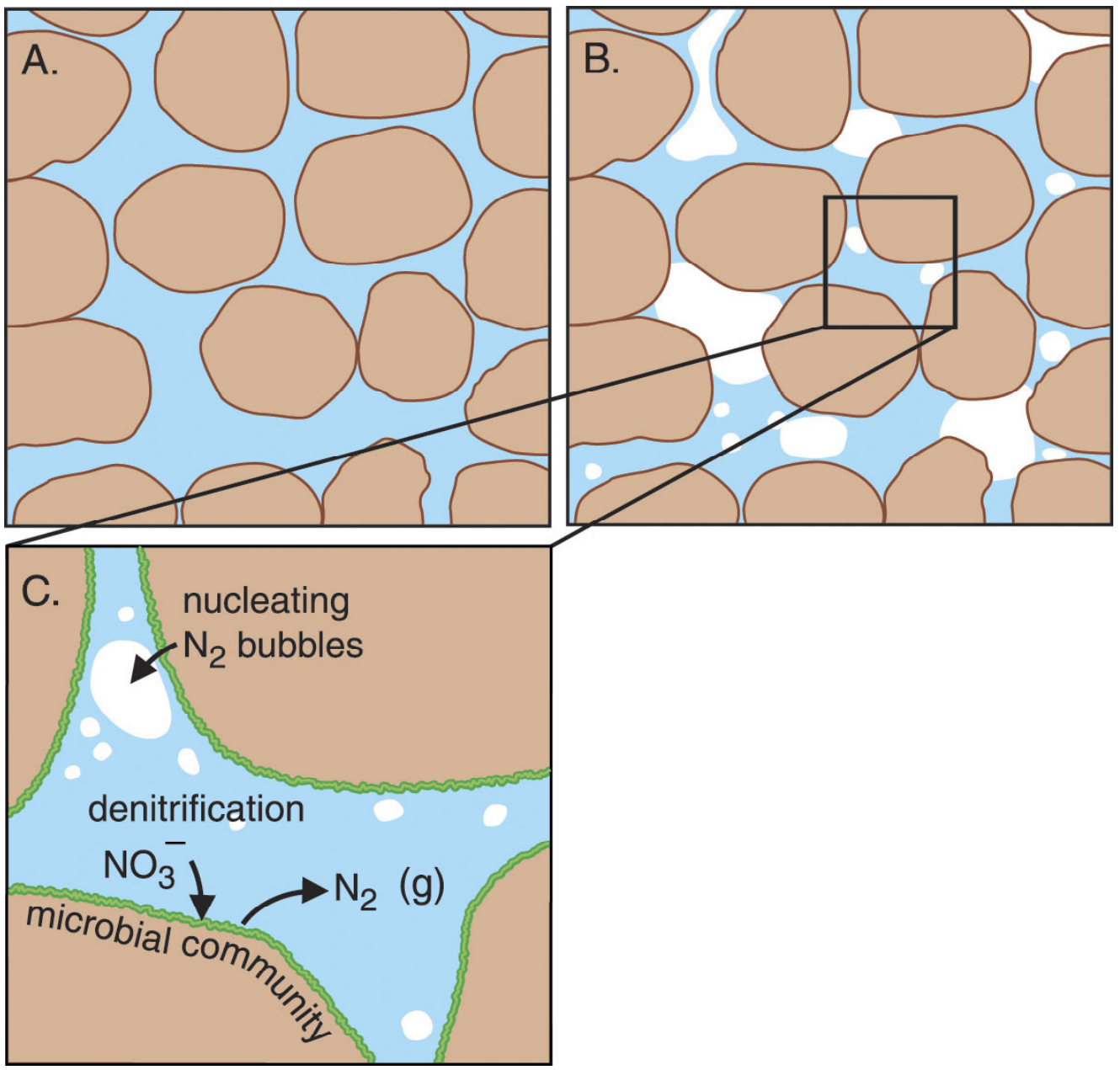

Figure 1.1 : Ilhustration show ing the idealized developm ent of respired gas bubbles $w$ ith in the pore spaces of saturated sedim ents. N ote the variety of bubble shapes that $m$ ay exist both $\mathrm{w}$ ith in and betw een pores. 
marine) subsurface environment where such an effect might be occurring. Aside from borehole fluid sampling, which is limited in its zone of investigation, there is presently no way to determine non-invasively whether in situ gas production is having potentially deleterious effects on the subsurface environment.

Ongoing research in the area of high-resolution marine geophysical imaging techniques suggested an alternative method for confirming the presence of isolated gas bubbles in the saturated subsurface environment. This is based on the principle that the propagation of high-frequency acoustic waves through saturated sediments is strongly affected by changes in the pore water gas phase concentration. The detailed study of shallow water gas-filled sediments in Eckernförde Bay, Germany and the interpretation of high-frequency marine acoustic profiling data acquired there provide a suitable framework upon which to evaluate the applicability of the aforementioned monitoring system.

In the Eckernförde Bay study, the research was aimed at improving the understanding of the benthic boundary layer processes that yield methane-rich sediments by utilizing high-frequency marine sonar data to better understand the dynamics of the trapped gas [Richardson and Davis, 1998]. Detailed laboratory studies had shown that even minute additions of gas to the saturated pore space $(<0.1 \%$ of the volume of the pore) result in substantial decreases in the amplitudes of transmitted 
acoustic waves [Anderson and Hampton, 1980a,b]. This is especially the case when then frequency of the transmitted wave closely matches the fundamental resonance frequency for bubbles of a given dimension [Anderson et al., 1998]. Thus, by recording the properties of acoustic waves interacting with both gassy and non-gassy sediments and modeling the expected effects, the researchers were able to match both the bubble sizes and depth of their occurrence [Lyons et al., 1996]. These results were later verified though sediment core recovery [Wilkins and Richardson, 1998].

A similar effect would therefore be expected for the transmission of acoustic waves through non-marine sediments in which redox conditions are favorable for the production of a variety of gaseous end products. These zones might include regions of denitrification $\left(\mathrm{N}_{2}\right)$, sulfate reduction $\left(\mathrm{H}_{2} \mathrm{~S}\right)$, methanogenesis $\left(\mathrm{CH}_{4}\right)$, or vigorous respiration $\left(\mathrm{CO}_{2}\right)$. From a monitoring standpoint, it should be possible to design a system to monitor gas production that relies on the repeated transmission of acoustic waves through a zone of interest. By monitoring any significant decreases in the amplitude of the transmitted acoustic waves over time, regions where the attenuating effect is occurring might be correlated with the in situ accumulations of biogenic gas. The general approach and layout of such a system is illustrated in Figure 1.2. 
A.

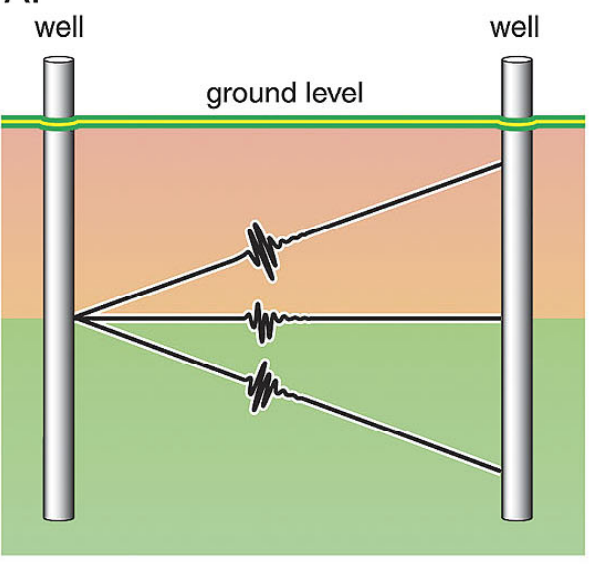

B.

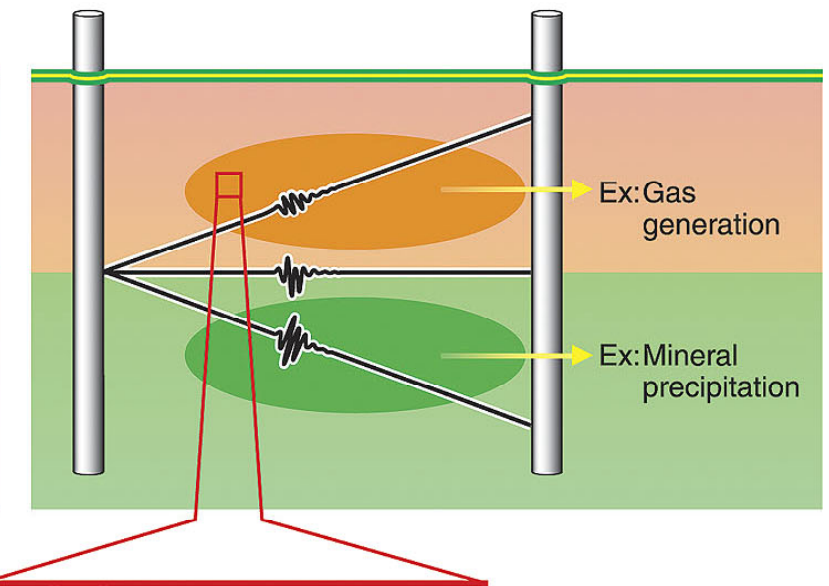

C.

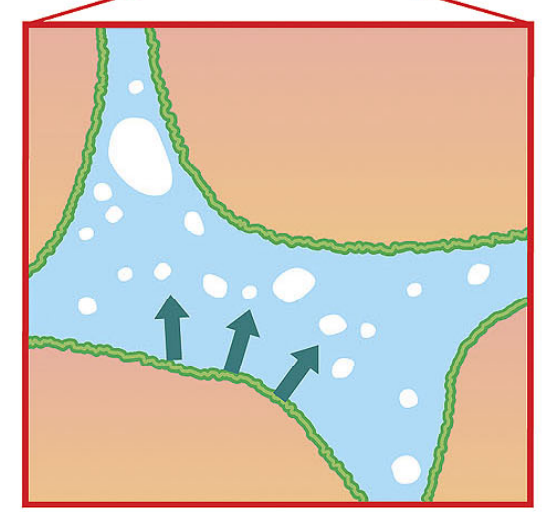

Figure 12: Ilhustration showing the potential layout of a cross-well geophysical system designed to $\mathrm{m}$ onitor terrestrial subsurface $\mathrm{m}$ icrobial activity over tim e. Only a few of the m any potential acoustic w ave paths are show $n$. N ote that changes in subsurface conditions m ay arise through a variety of $m$ eans, not solely gas production. A ttenuation of acoustic w ave energy through a region of gas production is show $n$ in $b$ ) for the conditions depicted in $\mathrm{c}$ ). 
The nature of such a method of detection allows for the investigation of regions outside the reach of more traditional hydrologic approaches; chief among these being water sampling and its reliance upon point measurements along a borehole. Furthermore, it extends the reach of the methodology laid out in the Eckernförde Bay study beyond the marine environment and into the terrestrial subsurface.

This experiment had as its goal the design of such a system and the successful integration of both traditional microbiological and hydrological methods with the more novel geophysical approach. The resulting thesis documents the laboratory and analytical procedures developed to test the aforementioned concepts under tightly controlled laboratory conditions. Furthermore, it provides the framework upon which a field-deployable system might be designed and put into use as a subsurface microbiological monitoring system: a so-called Microbial Megascope. 


\section{Chapter 2}

\section{Experimental Approach and Methods}

\subsection{Introduction}

The overall approach of the experiment was to show that microbial processes in the subsurface create physical property changes that can be detected with geophysical methods. In the case of denitrification, the primary change is the production and accumulation of microbe generated dinitrogen within the pore spaces of saturated sediments.

The objectives of the experiment were multifold: a) to determine whether denitrifying bacteria were capable of producing gaseous $\mathrm{N}_{2}$ in sufficient quantity to result in pore throat blocking; b) to measure any decrease in $\mathrm{K}_{\mathrm{s}}$ within the sediment column as a function of time and gas generation; c) to correlate the presence of zones of low acoustic wave 
amplitude with regions of high gas content; and d) to determine the distribution and cell density of denitrifying microbes within the column over the duration of the experiment.

\subsection{Assimilatory and Dissimilatory Half Reactions}

Prior to the onset of the experimental work, an effort was made to estimate the stoichiometric half-reactions which would govern the conditions within the sediment columns. The approach taken was to assume that only conditions favoring denitrification would exist within the columns (e.g. depleted oxygen concentrations, maintenance of suboxic conditions, sufficient nitrate availability, etc.)

More specifically, the microorganisms under investigation would be assumed to grow anaerobically on an organic carbon substrate (acetate; $\mathrm{CH}_{3} \mathrm{COO}^{-}$) while using nitrate for assimilatory cell synthesis with an efficiency of $20 \%$. The remaining $80 \%$ of the nitrate was presumed to be used exclusively for dissimilatory (i.e. energy generating) nitrate reduction in the form of denitrification. The resulting stoichiometric halfreactions were determined as per the method outlined in numerous texts covering environmental microbiology and reflect the aforementioned assimilatory/dissimilatory fractionation [Nazaroff and Alvarez-Cohen, 2001, Rittmann and McCarty, 2001]. 


$$
\begin{gathered}
0.125 \mathrm{CH}_{3} \mathrm{COO}^{-}+0.25 \mathrm{H}_{2} 0 \rightarrow 0.25 \mathrm{CO}_{2}+0.875 \mathrm{H}^{+}+\mathrm{e}^{-} \\
0.16 \mathrm{NO}_{3}^{-}+0.96 \mathrm{H}^{+}+0.8 \mathrm{e}^{-} \rightarrow 0.08 \mathrm{~N}_{2}+0.48 \mathrm{H}_{2} \mathrm{O} \\
0.036 \mathrm{CO}_{2}+0.007 \mathrm{NO}_{3}^{-}+0.207 \mathrm{H}^{+}+0.2 \mathrm{e}^{-} \rightarrow 0.007 \mathrm{C}_{5} \mathrm{H}_{7} \mathrm{O}_{2} \mathrm{~N}+0.079 \mathrm{H}_{2} \mathrm{O}
\end{gathered}
$$

$0.125 \mathrm{CH}_{3} \mathrm{COO}^{-}+0.167 \mathrm{NO}_{3}^{-}+0.292 \mathrm{H}^{+} \rightarrow 0.007 \mathrm{C}_{5} \mathrm{H}_{7} \mathrm{O}_{2} \mathrm{~N}+0.08 \mathrm{~N}_{2}+0.309 \mathrm{H}_{2} \mathrm{O}+0.214 \mathrm{CO}_{2}$

The resulting stoichiometric coefficients from the balanced overall reaction may then be used to make predictions regarding the quantity of acetate consumed or the amount of dinitrogen or cellular biomass produced. Such predictions may then be compared to the observed values and used to assess the suitability of the governing half reactions. Any deviations from these predictions may be useful in designing future experiments or interpreting anomalous observations.

\subsection{Laboratory Studies}

All of the experimental work was conducted within polycarbonate sediment columns of the type manufactured by Soil Measurement Systems, Inc. and shown in Figure 2.1. The flow cells or columns allow for a sealed environment into which a variety of wetting fluids may be introduced to a porous media and subsequently sealed in an air-tight fashion. Furthermore, the expectation of produced gases required that the design be able to accommodate any increases in pressure occurring over 


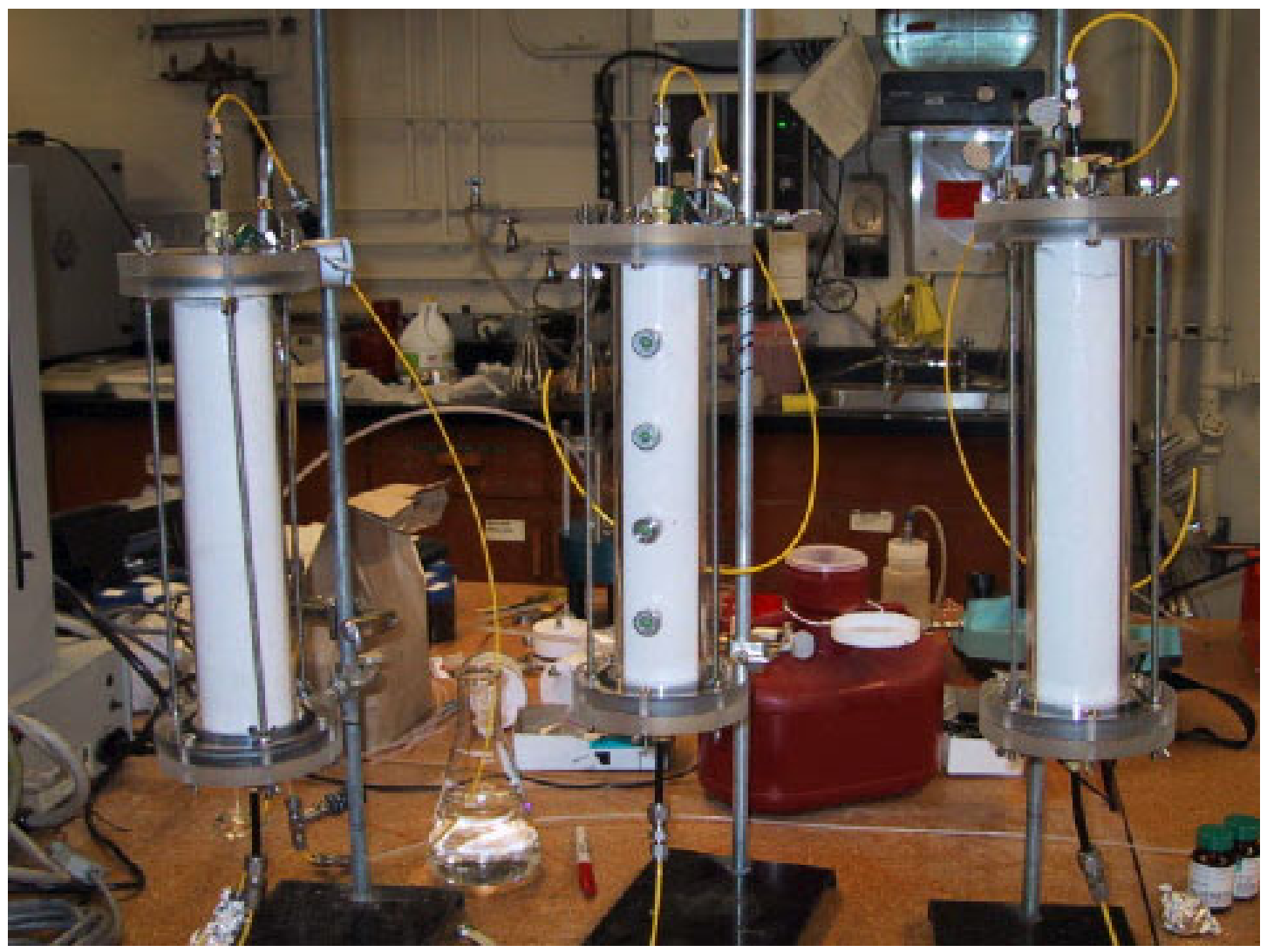

F igure 2.1: Photograph show ing three of the colum ns used in the experim ent and the generaldesign of each. The features shared by all of the colum ns are clear Polycarbonate cham bers, gas and w ater tight seals, rem ovable end caps, and adjustable influent and effluent lines. 
the duration of the experiment. Previous work by researchers in this area [Delozada et. al., 1994] suggested that the chosen design would allow for both easy modification of any necessary parameters as well as visual access to any changing column conditions. Details of the column design shall be described below (Section 2.3.3 Saturation of the Columns and Nutrient Addition) with the relevant dimensions included in Appendix A.

\subsubsection{Sediment Selection}

Silica sand of uniform grain size and physical properties was selected based upon its previous use in high-frequency acoustic wave propagation experiments of a similar scale [Meyer and Nakagawa, 2000]. The sand (U.S. Silica F-60 unground silica, Ottawa, Illinois) was oven dried to determine the bulk density. Using this value, the porosity was determined, as was the pore volume of a sediment-packed column. These values as well as other pertinent details concerning the sand are included in Appendix A. The sand was autoclaved prior to starting the experiment to destroy any unwanted microbes before inoculation with the known denitrifying species.

\subsubsection{Microbial Growth and Inoculation}

The denitrifying microbe chosen was Pseudomonas stutzeri (strain KC) as it was known to carry the reduction of nitrate all the way to dinitrogen 
[Madigan et al., 2000]. This was an important consideration as the desired outcome of the experiment was predicated on a gaseous product. Simplicity argued that the reduction of nitrogen be carried out by only one microbial strain; however, a consortium of microbes facilitating the following reduction sequence [Myrold, 1999] would have yielded similar results.

$$
\mathrm{NO}_{3}{ }^{-} \rightarrow \mathrm{NO}_{2}{ }^{-} \rightarrow \mathrm{NO} \rightarrow \mathrm{N}_{2} \mathrm{O} \rightarrow \mathrm{N}_{2}
$$

Given the high solubilities of both nitric and nitrous oxides relative to dinitrogen, these species were not desirable products. The fact that $P$. stutzeri alone was capable of yielding dinitrogen through the process of denitrification meant that only one strain had to be handled in inoculating the sediment columns. In this way, the tasks surrounding the growth and inoculation of the microbes was greatly simplified over that of working with a more complex microbial consortium and the inherent difficulties in accurately determining its precise community composition.

The selected strain was provided courtesy of Dr. Fred Brockman at the Pacific Northwest National Laboratory (PNNL). It was sourced from a streaked R2A agar plate having large numbers of actively growing colony forming units (CFU) with the characteristic morphology indicative of $P$. stutzeri. In a sterile laminar flow hood, a flame-sterilized loop was filled with a portion of one of the colonies. It was then added to a flask 
containing a complex medium comprised of dilute tryptic soy broth (TSB at $10 \%$ dilution) and allowed to grow up over a period of several days. The flask containing the inoculum was placed within an incubator/shaker set to $30^{\circ} \mathrm{C}$ and 50 revolutions per minute. After a period of two days, stationary growth phase was reached with a cell concentration in the TSB solution taken to be $\sim 1 \times 10^{9}$ cells $/ \mathrm{ml}$.

Previous work utilizing $P$. stutzeri indicated that growth on dilute TSB greatly reduced the degree of cell clumping in the inoculating solution [Fred Brockman, personal communication]. It was hoped that reduced cell clumping would result in a more uniform sediment inoculation. As a uniform cell distribution was desired, dilute TSB was chosen over the defined medium (100x Medium D) utilized once the columns were packed and saturated. The choice of a defined rather than a complex medium was made to ensure that conditions for denitrification were optimized and that unwanted growth by contaminating strains would be minimized.

At this point, $1 \mathrm{ml}$ of the cell suspension was added to each of three $30 \mathrm{~mL}$ centrifuge tubes with each tube representing the starting cell concentration for a given sediment column. The tubes were then filled with $19 \mathrm{~mL}$ of the dilute TSB media and again allowed to grow to stationary phase within the incubator/shaker yielding a total of $\sim 2.0 \times 10^{10}$ cells/tube. The rationale behind this particular concentration was based 
upon the desired starting cell concentrations within each sediment column and the expected losses due to fluid flow during column saturation.

Prior work involving fluid flow through microcosms suggested that a reasonable estimate for cell attachment efficiency during saturation would be approximately 25\% [Brockman, personal communication]. In other words, it was assumed that only $25 \%$ of the cells would remain affixed to the mineral grains after saturation and flushing of the columns was completed.

The tubes were then centrifuged in a Beckman Avanti J-25 centrifuge at $8000 \mathrm{x}$ g for 20 minutes. The supernatant was removed with a sterile pipette and $20 \mathrm{~mL}$ of phosphate buffer was added to each the centrifuge tubes. The cells were then re-suspended by vortexing each of the tubes. These two steps were then repeated in order to remove any trace amounts of nutrients that may have remained.

The cells were then added to a suspending solution of $250 \mathrm{~mL}$ of filter sterilized defined medium that had been pre-selected for growing denitrifying bacteria. The exact composition of the defined medium is included in Appendix A. The $250 \mathrm{~mL}$ fluid volume corresponded to roughly one third of the total pore volume of the sediment columns (see Appendix A) and was chosen to promote sufficient but not undue sediment saturation. To avoid premature growth, the defined media used for the 
cell suspension included neither the carbon source (acetate) nor the terminal electron acceptor (nitrate).

The microbe-infused solution was then added to the autoclaved sand and mixed thoroughly with a sterile wand to promote a uniform distribution of microbes throughout the sand. The inoculated sand was set aside for a period of three days to allow for sufficient cell-sand grain adhesion before packing it into the sediment columns. It was feared that insufficient adhesion would result in a significant loss of microbes during saturation and that the chance of promoting the desired effect would be accordingly diminished.

It should be pointed out that the cells were allowed to adhere within a large autoclaved tray placed within a laminar flow hood to prevent contamination. This resulted in a fortuitous condition in which the constant airflow within the hood partially desiccated the cell-sand mixture. The desiccated mixture allowed a much more thorough saturation procedure to be followed and thus allowed for the removal of the vast bulk of the entrained air. Subsequent experimental work with wet or partially saturated sediments has resulted in rather poor displacement of entrapped air bubbles with the repercussions for unimpeded acoustic wave propagation experiments being apparent.

As a reminder, a starting condition of complete sediment saturation within the columns was desired in order to assess the magnitude of 
decreasing acoustic wave amplitudes as gas was produced in the pore space over time. What was not well established at the time of the experiment, however, was the desiccation tolerance of $P$. stutzeri and its ability to withstand first drying and then re-wetting during column saturation. As shall be seen, the fears of cell death due to desiccation and/or lysing during re-wetting were unwarranted. It appears that the cells were able to survive both conditions, no doubt existing within a thin fluid film coating each mineral grain.

\subsubsection{Saturation of the Columns and Nutrient Addition}

The inoculated sand was then transferred to four specially designed columns and packed in the manner described in Fry et al. [1997]. One column was used for gas sampling, the second was used to measure $K_{s}$, the third was used to make the cross-column acoustic measurements, and the fourth was used as the control column. Each of the columns was fitted with end plates incorporating valves connecting the column to a fluid source and an adjustable outlet pipe. The columns were then completely saturated in such as way as to minimize any trapped gas within the pore spaces. As the effect of such gas was what was to be measured, the goal was to ensure that all of pore spaces were fluid-filled before starting. The methods used for ensuring near total saturation have been described in Faybeshenko [1995] and Fry et al. [1997]. 
Briefly, the columns were flushed with several pore volumes of $\mathrm{CO}_{2}$ before both de-aired phosphate buffer and the growth medium was introduced from the base of the column. The introduction of pure $\mathrm{CO}_{2}$ had the advantage of both displacing the air occupying the pore space due to its greater density as well as being extremely soluble once saturated. The flow of $\mathrm{CO}_{2}$ through the majority of the pore spaces in the column would have been greatly impeded had the sediment not been partially desiccated. The flow was regulated using a Gilmont Instruments, Inc. micro-ruby ball flowmeter that provided an estimate of the rate of gas flowing through the column. A value of $9.5 \mathrm{ml} \mathrm{CO}_{2}$ per minute was chosen, resulting in one pore volume being flushed through the sediment column every 24 minutes. The various components are shown in relation to one of the columns in Figure 2.2.

As stated, several pore volumes of de-aired phosphate buffer were then run through the columns. As compared to the solute-rich defined medium, it was thought the lower ionic strength phosphate buffer would be less likely to lyse the cells upon saturation of the dry sediment columns. This was then followed by the addition of two pore volumes of de-aired growth medium with all of the saturating solutions being de-aired via vacuum extraction. Both saturating fluids were introduced to columns from the bottom at very slow rates of roughly $1 \mathrm{~mL} /$ minute. This was done to avoid any air entrainment resulting from top-down flow and to allow a thorough 


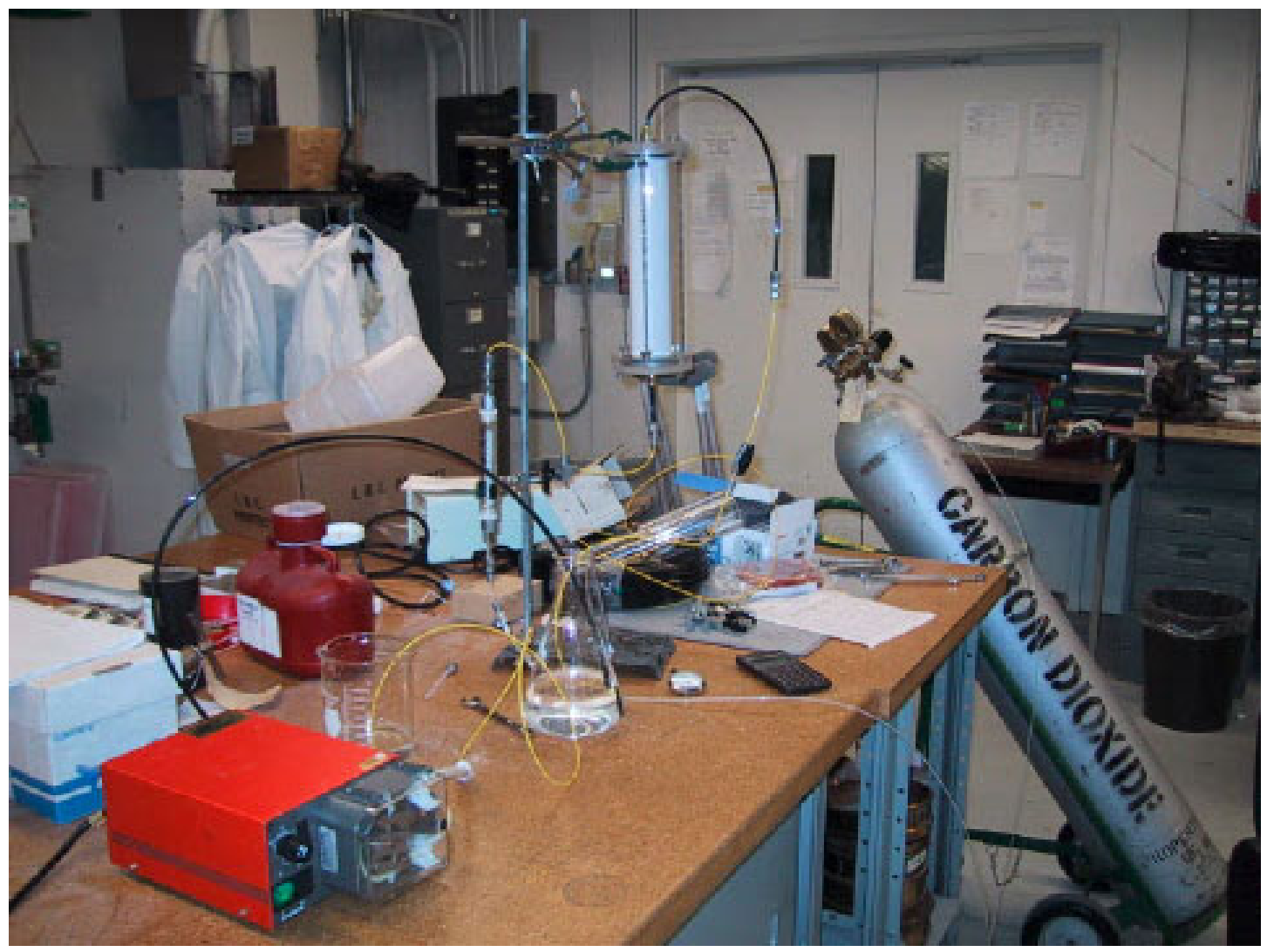

Figure 2 2: Photograph show ing the general arrangem ent of the carbon dioxide flushing system. Gas flowed from the cylinder through a calibrated flow indicator ( $m$ ounted on the ring stand to the low er left of the colum $\mathrm{n}$ ) and upw ard through the colum $\mathrm{n}$ displacing the less dense am bient pore space air. 
and pervasive wetting front to develop throughout the column. The rationale behind the introduction of several pore volumes of saturating solutions was to ensure the complete dissolution and removal of any remaining trapped $\mathrm{CO}_{2}$ in the pore spaces.

The defined growth media used for each of the columns was essentially identical for each column (recipe included in Appendix A). The only difference being that that the $\mathrm{NaNO}_{3}$ used for the gas-sampling column consisted of a $98 \%$ pure ${ }^{15} \mathrm{~N}$ labeled product obtained from Cambridge Isotope Laboratories, Inc. After adding the growth solution, the influent and effluent valves of the columns were shut and the columns incubated at room temperature $\left(\sim 25^{\circ} \mathrm{C}\right)$. It should be pointed out that as the appropriate equipment to allow for very low flow rates through the column were not available, no column flow occurred over the course of the experiment (other than during the measurement of $\mathrm{K}_{\mathrm{s}}$ ). This was justified in the sense that the outcome of the acoustic wave response was unknown at the onset of the experiment. By removing the possibility of entrapped gas loss due to flow, one less variable in interpreting the acoustic wave measurements was achieved.

At this time a fourth sediment column designated as the control column was also prepared. It was prepared in the same way as the other three columns although it contained only autoclaved, non-inoculated sediments. The saturating fluids and procedure were the same as those 
described above. The objective of the control column was to assess the potential for any chemically mediated (i.e. abiotic) processes leading to similar observable effects, such as decreased hydraulic conductivity and/or decreased acoustic wave amplitudes.

\subsubsection{Hydraulic Conductivity Measurements}

The measurements of $\mathrm{K}_{\mathrm{s}}$ were made using the constant head method described in Jury et al. [1991]. Briefly, the procedure involved the use of a Mariotte bottle whose outflow was connected to the inlet valve on the bottom of the column. This setup allowed for upward flow through the column, which was desirable as it decreased the likelihood that any gas collecting in the headspace would become entrained in the flow and be introduced to the pore space. $\mathrm{K}_{\mathrm{s}}$ was determined from a variation of Darcy's law. That is:

$$
\mathrm{K}_{\mathrm{s}}=\frac{\mathrm{V} \cdot \mathrm{L}}{\mathrm{A} \cdot \mathrm{t} \cdot \Delta \mathrm{h}}
$$

where $\mathrm{V}$ is the volume of growth medium discharging in time $\mathrm{t}, \mathrm{L}$ is the length of the sample $(28.5 \mathrm{~cm}), \mathrm{A}$ is the cross-sectional area of the sample $\left(20.27 \mathrm{~cm}^{2}\right)$, and $\Delta \mathrm{h}$ is the head difference between the inlet and the outlet $(5.7 \mathrm{~cm})$. The principle at work here is that the pressure inside of the Mariotte bottle at the level of the bubble tube is at atmospheric value. This maintains the head level on the inflow end of the sediment column at 
the same height as the end of the bubble tube such that the $\Delta \mathrm{h}$ value may be increased or decreased by simply raising or lowering the bottle. An image of the configuration used is included as Figure 2.3.

The nature of the Mariotte bottle used here allowed for a volume of growth medium sufficient for the duration of the experiment to be isolated within the fluid reservoir and kept sterile. This was critical, as the inflow of the medium was not intended to be a point of entry for contaminating microbes. $\mathrm{K}_{\mathrm{s}}$ values were reported as a function of time. After the measurement of $\mathrm{K}_{\mathrm{s}}$ was made, the column was put aside until the next data-sampling period with the column inflow and outflow valves shut.

\subsubsection{Gas Sampling Measurements}

Based on the experiences of Reynolds et al. [1992] and Beckwith et al. [2001], gas sampling of the sediment column and measurements of $\mathrm{K}_{\mathrm{S}}$ were both started during the anticipated lag period ( $\sim 4$ days). These measurements formed the basis for the background or starting conditions within the column. The gas samples were run on the isotope ratio mass spectrometer (IRMS) set to measure for atom $\%{ }^{15} \mathrm{~N}$. The threshold of detectability of ${ }^{15} \mathrm{~N}$ had been confirmed prior to initiation of the experiment [Don Herman, personal communication] and the amount of $\mathrm{Na}^{15} \mathrm{NO}_{3}$ added was more than sufficient to allow for gas generation of sufficient volume. 


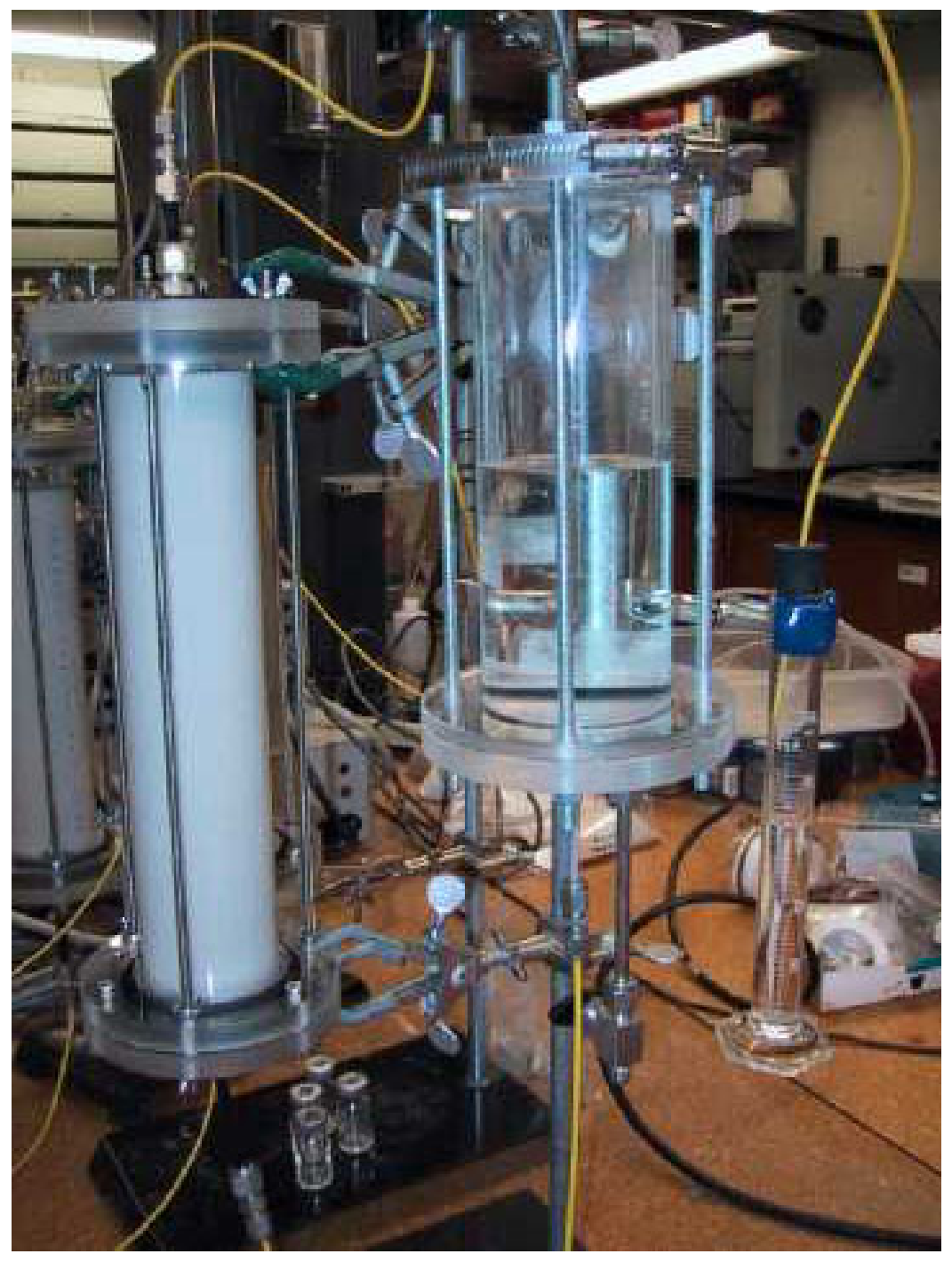

F igure 23 : Photograph show ing the assem bly used to m ake m easurem ents of saturated hydraulic conductivity. N ote the m odified M ariotte bottle m ounted on the ring stand above and to the right of the colum $\mathrm{n}$. The $\Delta \mathrm{h}$ value is determ ined by m easuring the distance from the bubble tube (inside the fluid reservoir) to the outflow (inside the graduated cylinder). 
Gas sampling occurred through the wall of one of the columns that had been outfitted with four separate gas permeation samplers of a type similar to those described in Beckwith et al. [2001]. In short, the design consisted of short segments of sealed, low-grade silicon tubing that had been previously determined to be gas- but not fluid-permeable. The tubing sections were coupled to barbed connectors that were terminated in septum-sealed gas chromatograph fittings. The assembly is shown in Figure 2.4. The operating principal was that any gas produced in the column in excess of the partial pressure of the gas within the silicon tubing would then diffuse across the membrane and would be available for sampling. Additionally, the sections of tubing were filled with sterilized, coarse sand in order to reduce the volume of gas exchange necessary for equilibration. Gas was then sampled by inserting the needle from a lockable gas tight syringe through the septum whereupon it was either stored in a separate septa-sealed sampling vial or inserted directly into the sampling port of the IRMS. The reason for the change in the sample introduction methodology becomes apparent upon review of the data (see Section 3.2).

\subsubsection{Acoustic Wave Measurements}

The column dedicated for acoustic wave measurements was evaluated using the high-frequency transmission seismic method described in brief 


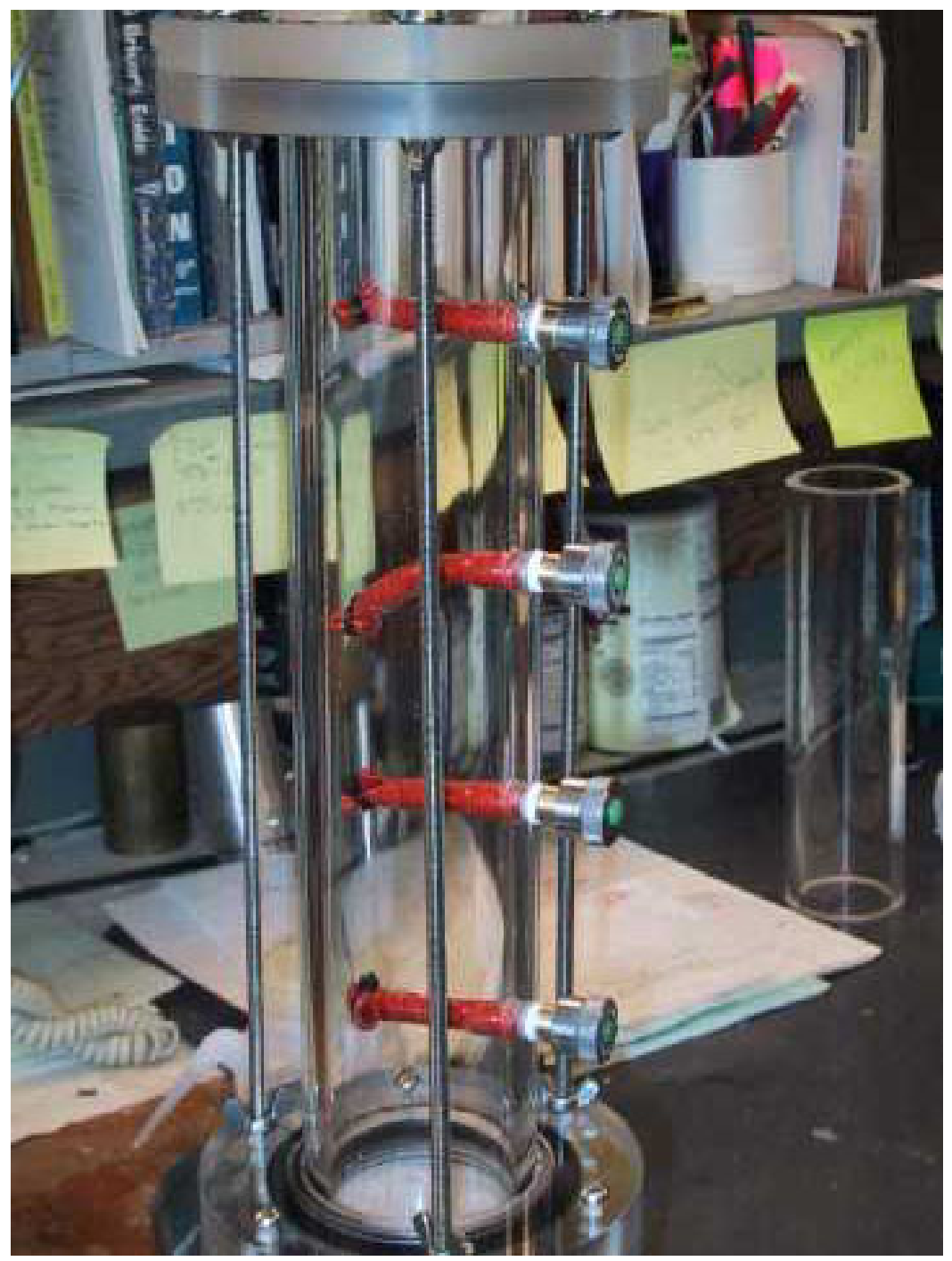

F igure 2.4 : Photograph show ing the assem bly used to extract gas sam ples from $\mathrm{w}$ ith in the colum $\mathrm{n}$. The gas perm eation sam plers $\mathrm{w}$ ere constructed of silicon tubing (red), inserted through the colum $\mathrm{n}$ wall, and connected to barbed gas chrom atograph septa valves (m etal/green). Gas diffused from the colum $n$ through the tubing and $w$ as $w$ ithdraw $n$ by syringe. 
earlier. The apparatus and methodology used here were similar to those described in Seifert et al. [1998] with some slight modifications made to accommodate the sediment column. The experimental design used here consisted of two Panametrics piezoelectric immersion transducers with a center frequency of $1 \mathrm{MHz}$ (Accusan-S and Accusan-R; Model Numbers A303S-Su and A303R-Su) being driven by the Panametrics Model 5058PR High Voltage Pulser-Receiver. The Model 5058PR is specifically designed for ultrasonic test and measurement applications that require a high material penetration capability. Given the diameter of the sediment column used here, this was a necessary feature.

A high frequency acoustic wave pulse was emitted from the piezoelectric source transducer and received by the transducer located on the opposite side of the column. In an attempt to promote reproducibility of the data, the seismic transducers were maintained in a fixed position within the walls of a large diameter tube filled with water. It was into this large water-filled tube that the column was lowered with the source and receiver transducers bracketing the column. The design is shown in Figures 2.5 and 2.6. The column was then raised and lowered within the water-filled tube and the measurements were made and recorded using a high-frequency oscilloscope (Tektronix Model TDS3012). The waveform characteristics of the seismic pulse and the time at which it was received were recorded digitally on the oscilloscope and transferred to personal 


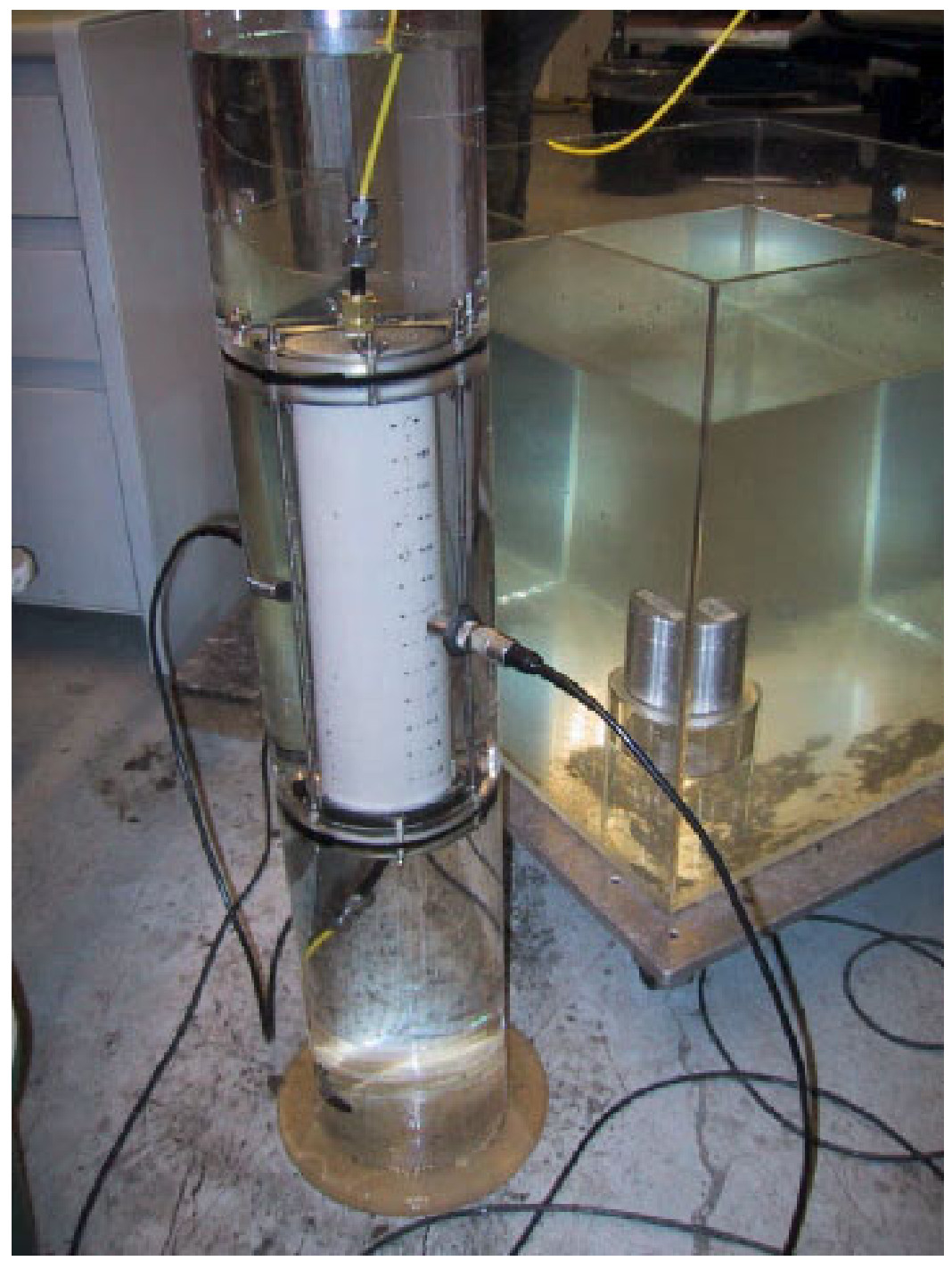

Figure 2 .5: Photograph show ing the apparatus designed to $\mathrm{m}$ ake acoustic $\mathrm{w}$ ave $\mathrm{m}$ easurem ents. The sedim ent colum $\mathrm{n}$ was inserted into a waterfilled tube into which the acoustic transducers were coupled. The transducers (piezoelectric cylinders $w$ ith black connecting cables) rem ain fixed while the colum $n$ w as raised/low ered $w$ ithin the tube (m easurem ent locations are visible). 


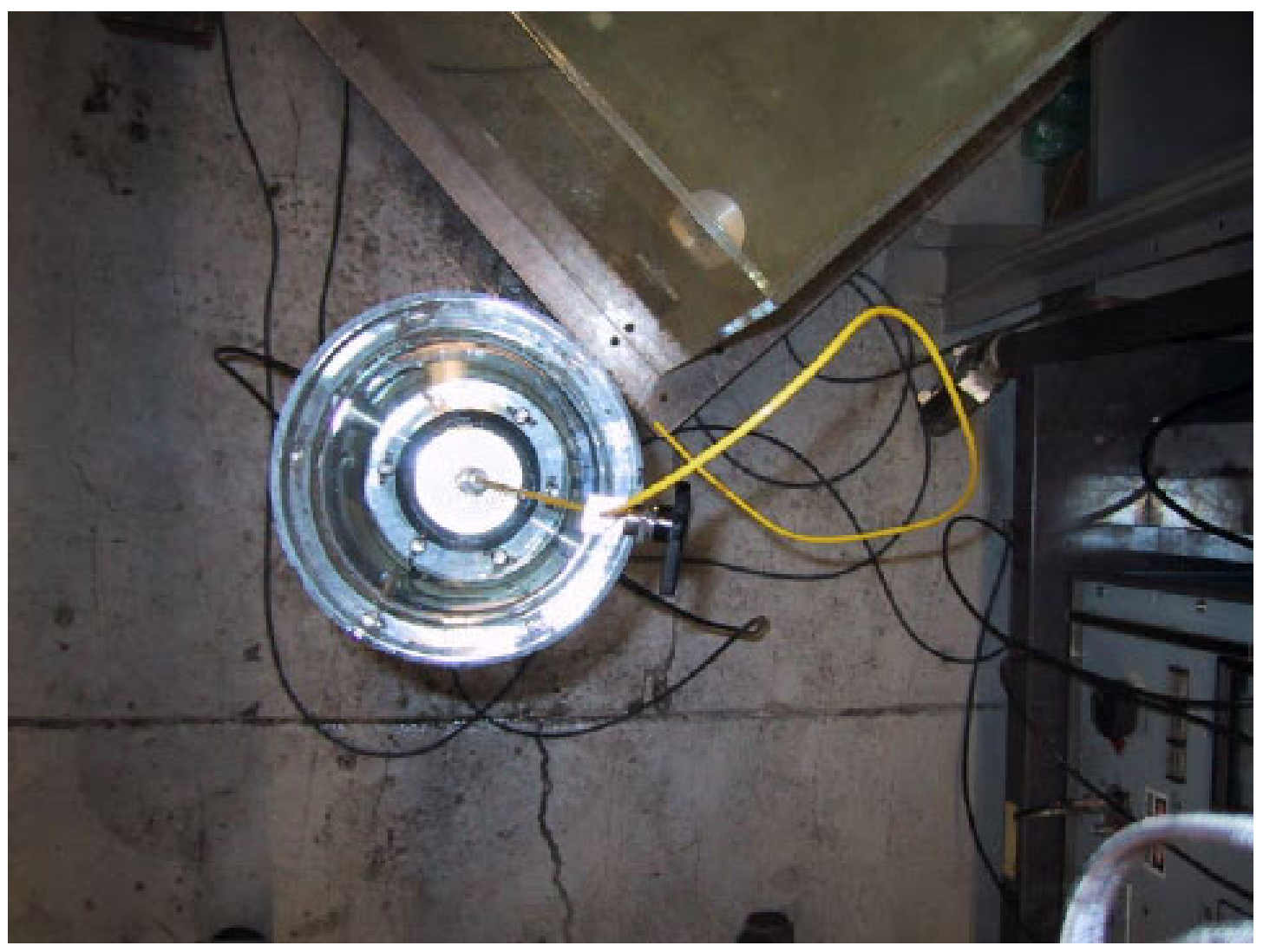

Figure 2 .6: Photograph show ing the apparatus designed to $\mathrm{m}$ ake acoustic $\mathrm{w}$ ave $\mathrm{m}$ easurem ents from the top. The $180^{\circ}$ alignm ent of the transducers is visible as is their offset from the colum $\mathrm{n} w$ all. 
computer for subsequent analysis. An initial set of measurements was made down the length of two of the columns (inoculated and control) in $1 \mathrm{~cm}$ intervals immediately after the addition of the growth solution. This was done in order to establish the baseline acoustic properties (e.g. wave amplitude, velocity, frequency and waveform characteristics). Subsequent measurements were made periodically as the growth of the microbes progressed. Once no further changes were observed in the columns, a final set of measurements was made.

\subsubsection{Microbial Biomass Estimation - Denitrification Assay}

Upon completion of the column experiments, one of the columns was chosen for destructive evaluation to assess the denitrifier biomass. The approximately $5 \mathrm{~cm}$ of water at the top of the seismic sediment column was removed as a sample (see Section 3.3 below), and the sand column was sectioned in a ring stand into 11 equally sized samples of approximately $2.5 \mathrm{~cm}$ thick by, when required, sawing through the polycarbonate liner. Samples were vortexed and aliquots of $10 \mathrm{~g}$ of sand removed and vortexed in $95 \mathrm{~mL}$ of $1 \%$ sodium pyrophosphate $(\mathrm{pH} 7)$. Serial dilutions were performed in phosphate buffered saline. Tubes containing R2A medium with $0.05 \%$ potassium nitrate were flushed with nitrogen gas to minimize oxygen levels, sealed, and autoclaved. One $\mathrm{ml}$ from the serial dilutions was injected into tubes in a 3 -tube most probable 
number (MPN) format. After 29 days incubation at room temperature, denitrification activity in each tube was colorimetrically assayed [Tiedje, 1982]. Absence of nitrate and/or presence of nitrite were considered positive for denitrification. 


\section{Chapter 3}

\section{Experimental Data and Results}

\subsection{Saturated Hydraulic Conductivity Measurements}

$\mathrm{K}_{\mathrm{s}}$ measurements were made on the dedicated column with the results shown in Figure 3.1. As can be seen, there is a drop in $\mathrm{K}_{\mathrm{s}}$ occurring roughly five days after the column was saturated. Also plotted is the data acquired for the control column over the same period. As can be seen, no measurable reduction in $\mathrm{K}_{\mathrm{s}}$ is observed.

When the drop in $K_{s}$ is considered more closely, a set of distinct patterns appear. The first few days following saturation coincides with an initial decrease in $\mathrm{K}_{\mathrm{s}}$ of $9.7 \%$. Throughout this period, no observable differences were recorded in the seismic column. Following this first reduction, there was a rather abrupt and marked decrease of $63.3 \%$ in $\mathrm{K}_{\mathrm{s}}$ 


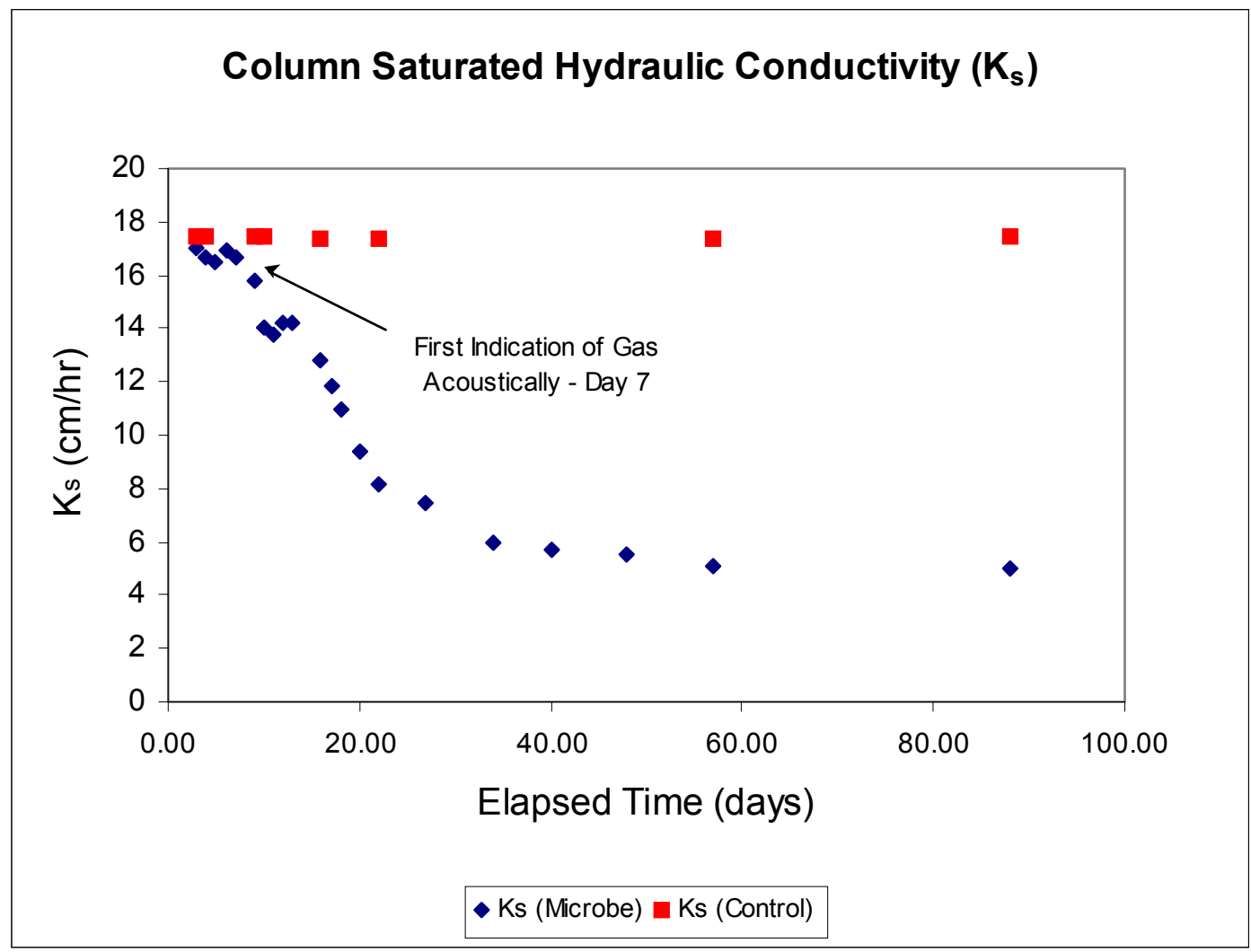

Figure 3.1: The results of the saturated hydraulic conductivity $\left(K_{s}\right)$ $\mathrm{m}$ easurem ents over the course of the experim ent. The first indication of gas $w$ ithin the acoustic $m$ on itoring colum $n$ is show for com parison. N ote that very little decrease in $\mathrm{K}_{\mathrm{s}}$ occurs before this tim e but that the values drop rapidly once gas appears, ultim ately stabilizing to a final value. The results from the control colum $n$ are also show $n$ for com parison. 
over the ensuing 30 days. During this time, dramatic decreases in acoustic wave amplitudes were observed matching the initial expectations of the experiment. There was very little measured decrease in $\mathrm{K}_{\mathrm{s}}$ after this time with the total decrease occurring since the onset of saturation amounting to $66.9 \%$. By this time, the evolved dinitrogen had been produced in sufficient quantity that large $(>2 \mathrm{~cm})$ accumulations of gas were directly observable along the column walls (Figure 3.2). After 90 days, the experimental objectives had been met and the measurements were halted.

Several scenarios exist for the early reduction in $\mathrm{K}_{\mathrm{s}}$ that did not correlate with any effect on acoustic wave propagation. One might include growth under microaerophilic conditions that could have existed initially if a small amount of dissolved oxygen were present in the growth solution. Conversely, the microbes might have been growing anaerobically but producing bubbles too small to be detected by the frequencies used during the acoustic wave measurements. Another possibility is that the initially produced dinitrogen was accommodated in the aqueous phase arising from the increased gas solubility as pressure rose within the sealed column. A better representation of this particular effect is illustrated in Section 3.3. 


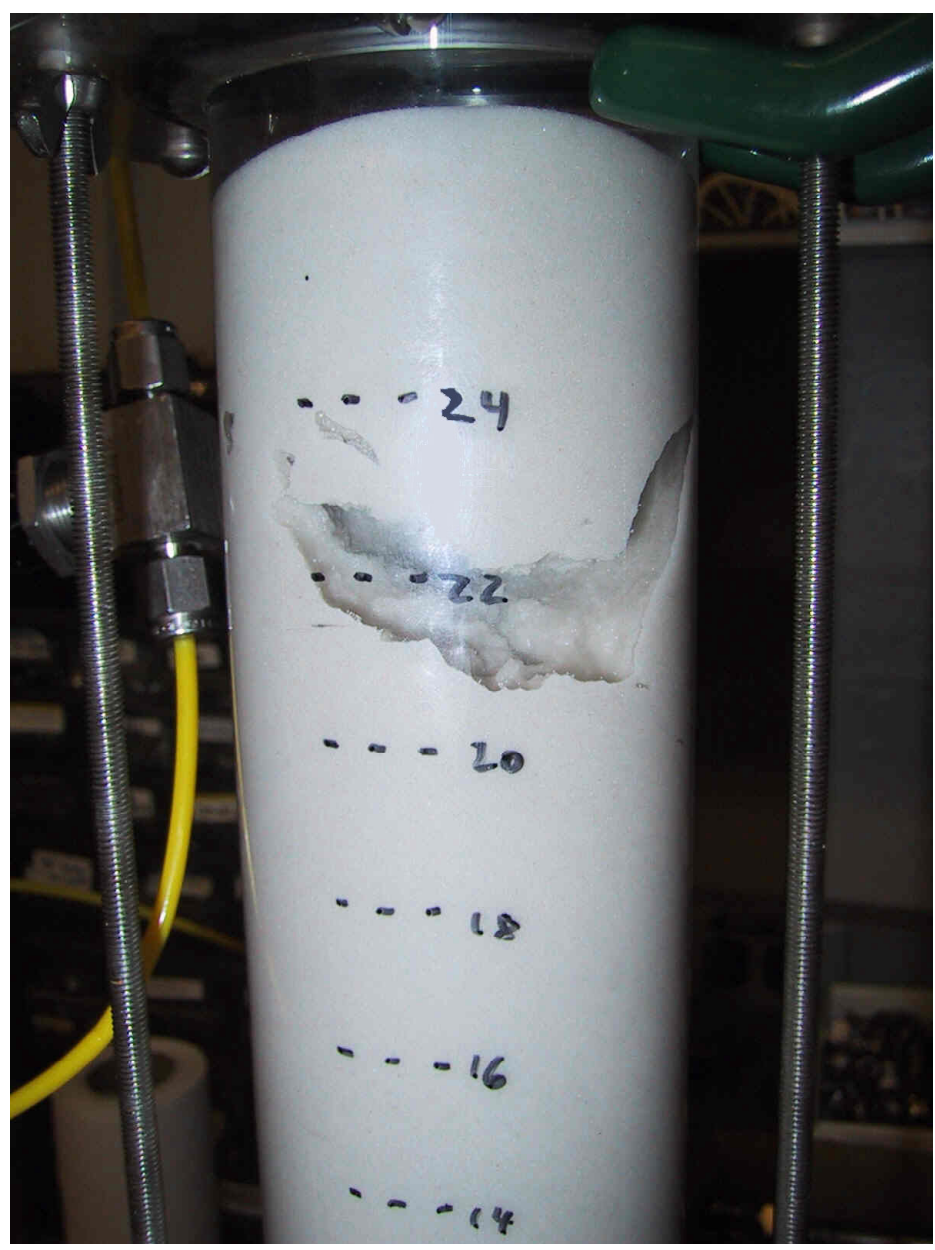

Figure 32 : C lose-up photograph of the acoustic m on itoring colum $n$ after 60 days have elapsed since saturation. The bubble accum ulation observed in this colum n w as sim ilar to that observed in the other inoculated colum ns. Its dim ensions were approxim ately $2 \mathrm{~cm} \times 3 \mathrm{~cm} \times 1 \mathrm{~cm}$ and it possibly represents entrapm ent and accum ulation along a packing discontinuity . 


\subsection{Atom $\%{ }^{15} \mathrm{~N}$ Gas Sampling}

The gas sampling had initially been envisioned to occur on a similar timescale to that of the $\mathrm{K}_{\mathrm{s}}$ sampling. Unfortunately, due to limited access to the IRMS, it was decided that over the course of the experiment only five representative samples would be collected and analyzed. The data are plotted in Figure 3.3 and the values included in Table 3.1. The data points shown include the fives sampling periods for each of four gas sampling ports and include both the replicate and averaged values. The ports are aligned along the length of the column with Port \#1 located near the top of the column and Port \#4 located near the bottom. Also included is the reference air sample that was run at the time of each analysis period.

Table 3.1: Atom $\%{ }^{15} \mathrm{~N}$ Values

\begin{tabular}{|ccccccc|}
\hline \multicolumn{2}{c}{$\begin{array}{c}1 \\
\text { Date }\end{array}$} & $\begin{array}{c}2 \\
\text { atom } \% 15 \mathrm{~N}\end{array}$ & $\begin{array}{c}3 \\
\text { atom } \% 15 \mathrm{~N}\end{array}$ & $\begin{array}{c}4 \\
\text { atom } \% 15 \mathrm{~N}\end{array}$ & $\begin{array}{c}\text { reference air } \\
\text { atom } \% 15 \mathrm{~N}\end{array}$ & $\begin{array}{c}\text { Elapsed } \\
\text { atom } \% 15 \mathrm{~N}\end{array}$ \\
\hline \hline Time (days)
\end{tabular}




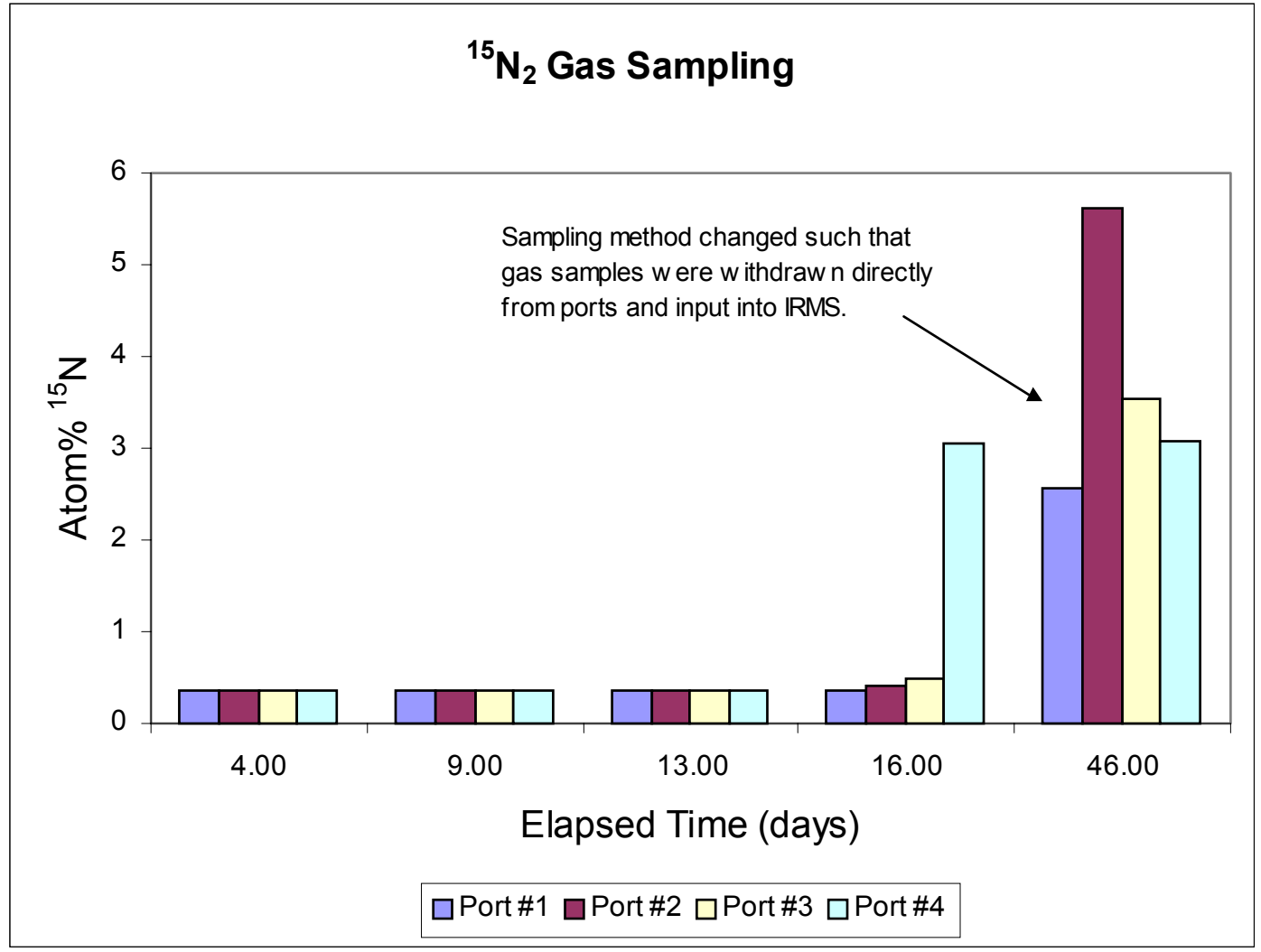

Figure 3.3 : The results of the isotope ratio m ass spectrom eter analysis of evolved colum $n{ }^{15} \mathrm{~N}$. The gas sam pled over the first two weeks of the experim ent show s no deviation from atm ospheric atom $\%{ }^{15} \mathrm{~N}$ (0.3663) but breakthrough of labeled gas appears to transpire once pressure $\mathrm{w}$ ith in the colum $n$ rises enough to enhance diffusion into the sam plers. The final results indicate that respired $\mathrm{N}_{2}$ was the only reasonable source of gas w ith in the colum ns. 
As can be seen, for three of the four ports there is only a very gentle increase in the sampled atom $\%{ }^{15} \mathrm{~N}$ over the first 16 days. In fact, the extracted air samples are indistinguishable in value from those of air (with a known atom $\%{ }^{15} \mathrm{~N}$ value of 0.3663 ). Port \#4, however, shows a dramatic increase in atom $\%{ }^{15} \mathrm{~N}$ sixteen days after initial column saturation. It is quite possible that the general lack of ${ }^{15} \mathrm{~N}_{2}$ collected and measured on the IRMS up to this point had to do with the gas permeation sampler design. The tests done before installation indicated that a rather large increase in gas partial pressure was necessary to drive gas across the sampler boundary. In other words, the ${ }^{15} \mathrm{~N}_{2}$ gas pressure within the column may not have been high enough for much of the experiment to force gas from the pore space into the samplers.

Another problem may have resulted from the procedure for gas sampling and transfer to the IRMS. Initially, the samples were removed from the permeation samplers via a gas-tight lockable syringe. The samples were then transferred to a septa-sealed vial before being transported to the IRMS. As only a $10 \mu \mathrm{L}$ sample was necessary for IRMS analysis, this resulted in a gross discrepancy between the $10 \mathrm{~mL}$ vial volume and the amount withdrawn from the permeation sampler. This volume discrepancy and perhaps a poor job of evacuating the vial prior to sample injection may have lead to the low values of atom $\%{ }^{15} \mathrm{~N}$. 
An opportunity became available to run a final set of samples on the IRMS 46 days after column saturation. By this time, sufficient evidence existed from the other measurement columns to suggest that the gas production was substantial enough to have overcome any partial pressure barrier to gas diffusion into the permeation samplers. This was confirmed through the observation of numerous gas bubbles through the wall of the gas-sampling column. The column was brought directly to the IRMS and the $10 \mu \mathrm{L}$ samples were removed from the permeation samplers and inserted directly into the IRMS circumventing any vial-related problems. The data values for this period illustrate the increased levels of atom $\%{ }^{15} \mathrm{~N}$. The gas being produced within the columns was clearly dinitrogen and in fact, the concentration of ${ }^{15} \mathrm{~N}$ was sufficiently large to saturate the IRMS settings for molecular mass 30 -analysis (i.e. ${ }^{15} \mathrm{~N}^{15} \mathrm{~N}$ ). The settings were adjusted to avoid peak area curve clipping per Atkins et al. [1992] and the corrected values are reported here.

\subsection{Acoustic Wave Measurements}

The acoustic wave measurements represent the focus of the experiment and they will be considered in more detail than the other sets of measurements. As mentioned briefly above, the column used to make these measurements was divided into a series of one-centimeter intervals down the length of the column. The largest of the values $(24 \mathrm{~cm})$ 
corresponds to the top of the column while the smallest value $(1 \mathrm{~cm})$ corresponds to the bottom.

After saturation of the column had been completed, it was noted that settling of the packed sand grains resulted in a fluid-filled headspace between the uppermost layer of sediment and the top of the column. This headspace provided a useful 'window' into which gas ebullition could be observed over the course of the experiment. The fluid-filled region was roughly $1-2 \mathrm{~cm}$ above the highest measurement point $(24 \mathrm{~cm})$ and its presence may account for some of the specific data features.

\subsubsection{Acoustic Waveform Data}

The acoustic waveform data for each location down the column as a function of time represents the primary form of data acquired. For the purposes of this thesis, four measurement locations shall be considered which sufficiently represent conditions throughout the column. These locations include the following depths: $24 \mathrm{~cm}, 16 \mathrm{~cm}, 8 \mathrm{~cm}$, and $4 \mathrm{~cm}$. The period over which the measurements were made is as follows: $3,7,11$, and 28 days elapsed since column saturation. The complete waveform data for each of these locations over time is presented in Figures 3.4 to 3.7. Also included for comparison is the waveform data collected at four locations for two periods within the control column (Figures 3.8 to 3.11). 

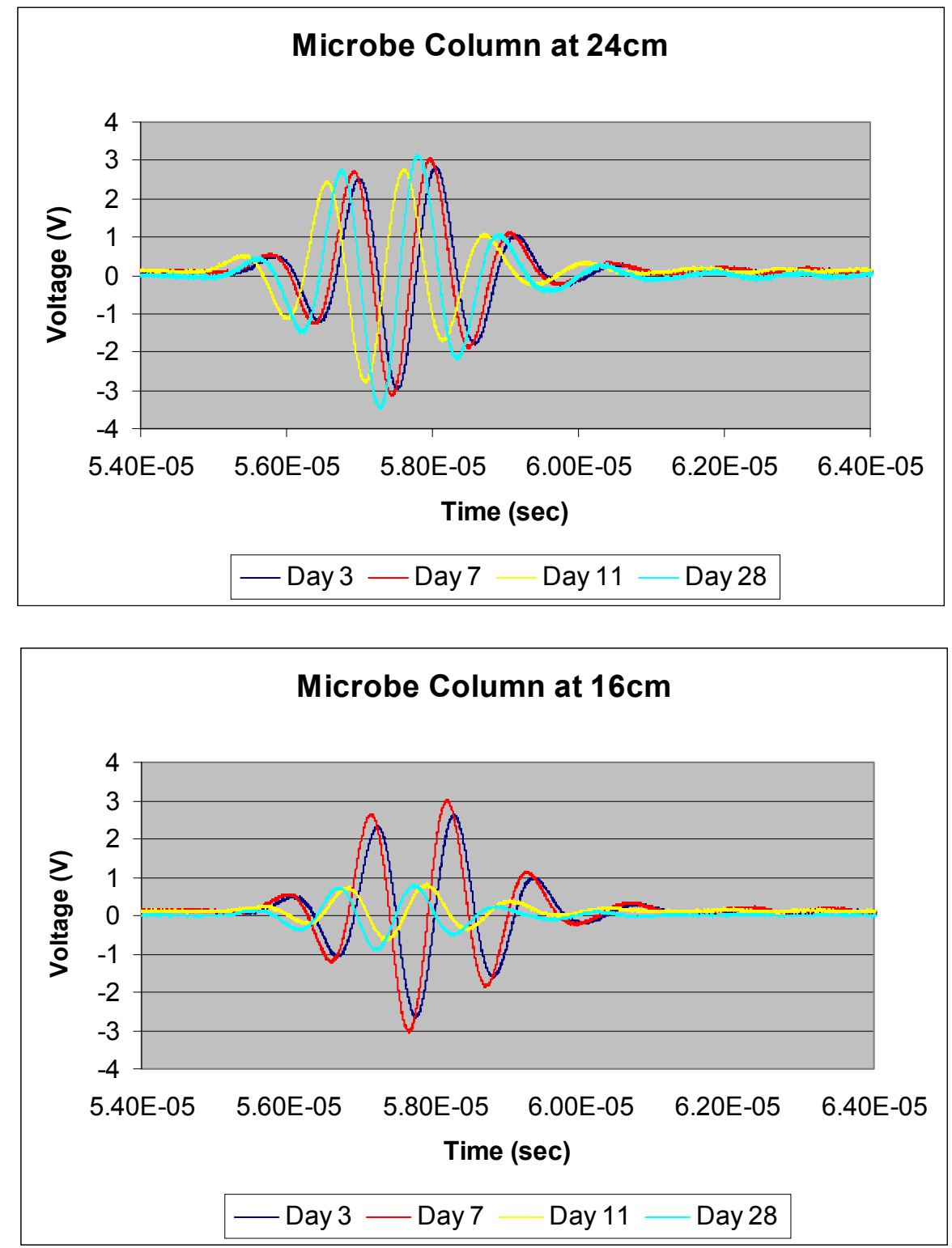

Figures 3.4 and 3.5: A coustic w aveform data from the m icrobe inoculated colum $\mathrm{n}$ for the $24 \mathrm{~cm}$ and $16 \mathrm{~cm}$ depth locations over four periods of tim e. $\mathrm{N}$ ote the travel tim e differences and am plitude decreases as a function of tim e and gas evolution. 

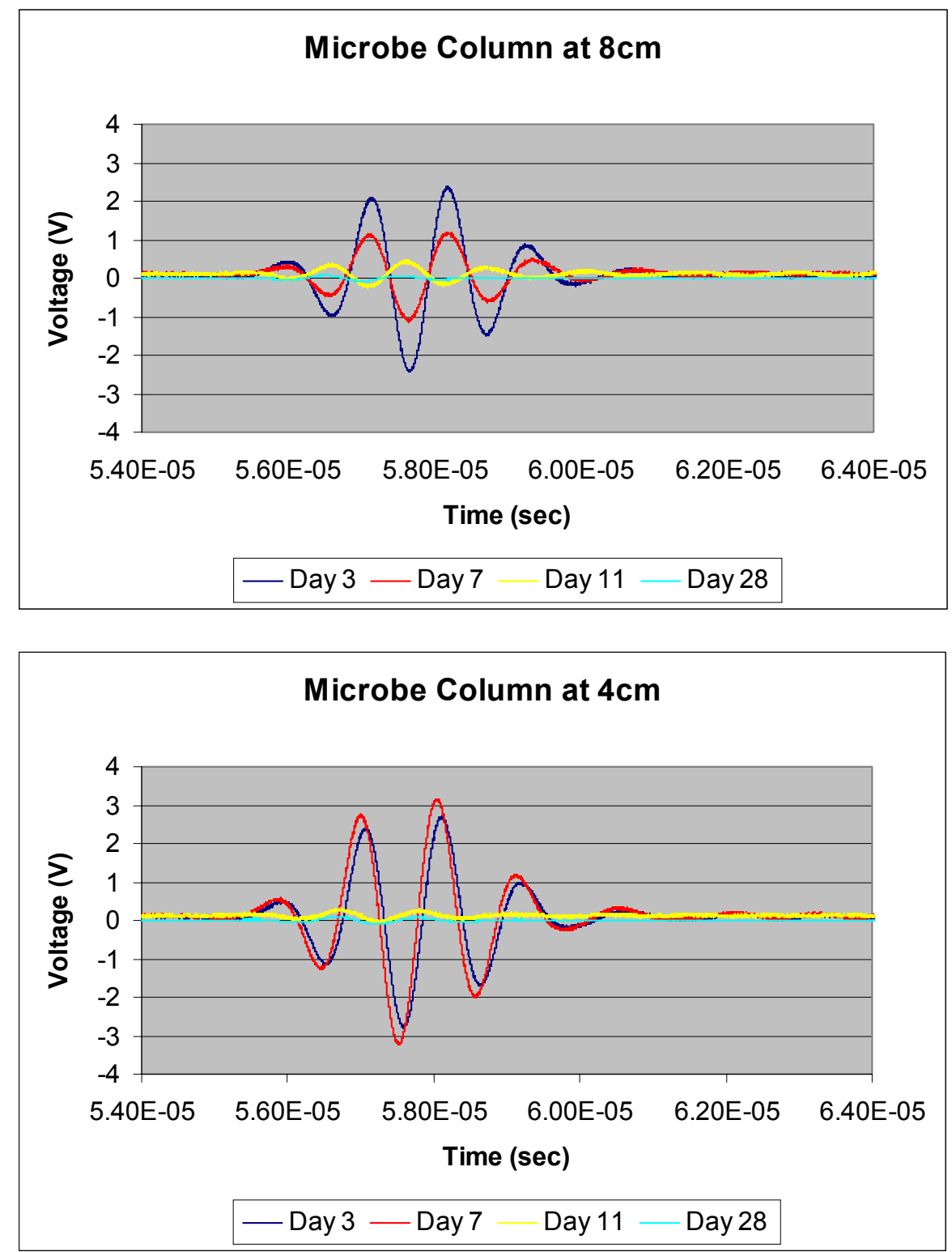

Figures 3.6 and 3.7: A coustic w aveform data from the m icrobe inoculated colum $\mathrm{n}$ for the $8 \mathrm{~cm}$ and $4 \mathrm{~cm}$ depth locations over four periods of tim e. $\mathrm{N}$ ote the travel tim e differences and am plitude decreases as a function of tim e and gas evolution. 

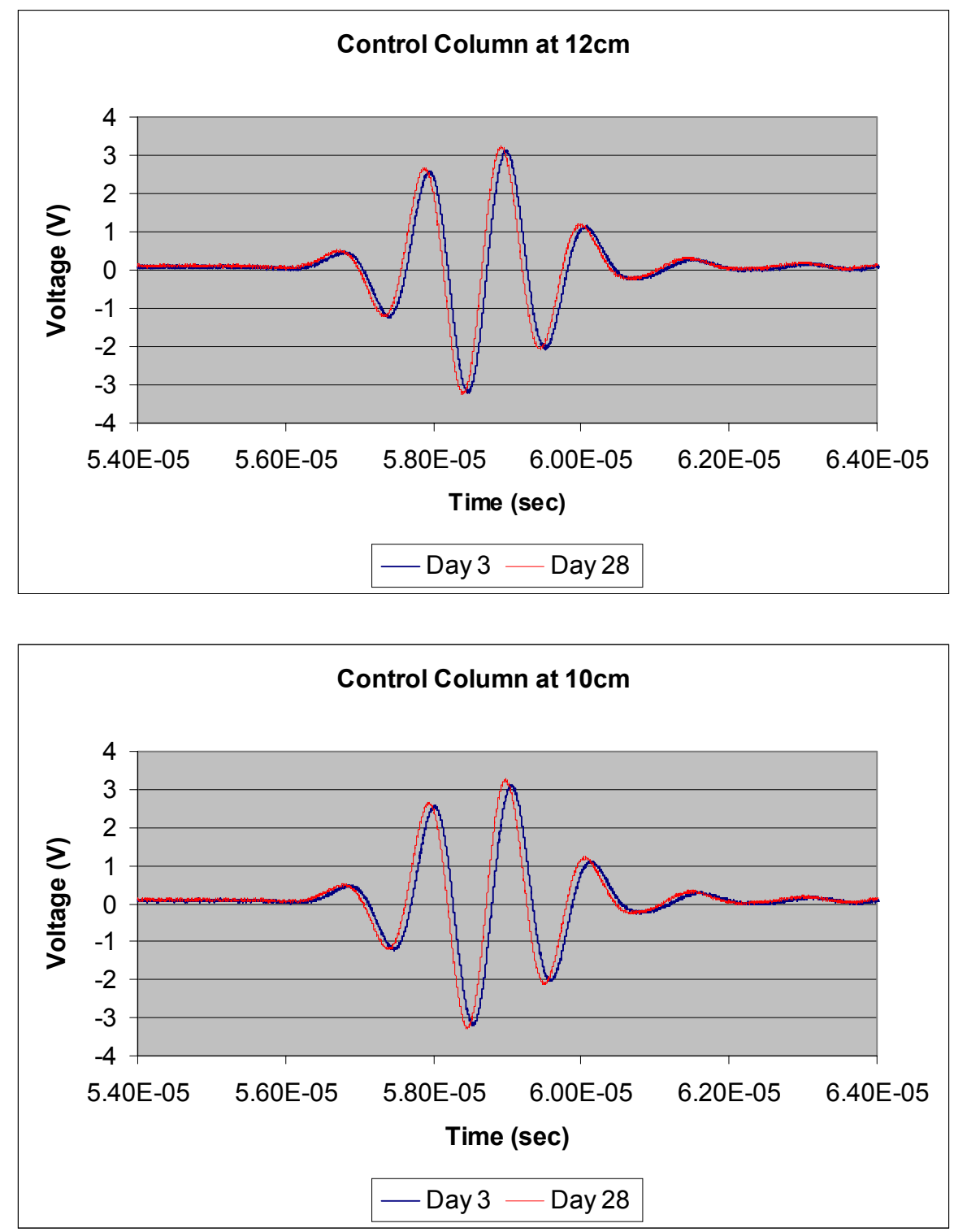

Figures 3.8 and 3.9: A coustic w aveform data from the control colum $n$ for the $12 \mathrm{~cm}$ and $10 \mathrm{~cm}$ depth locations over tw 0 periods of tim e. N ote the high degree of repeatability of the w aveform s. Such repeatability suggests that the effects observed in the inoculated colum $n$ are exclusively biogen ic in origin as the control colum $n$ received the sam e saturating m edium . 

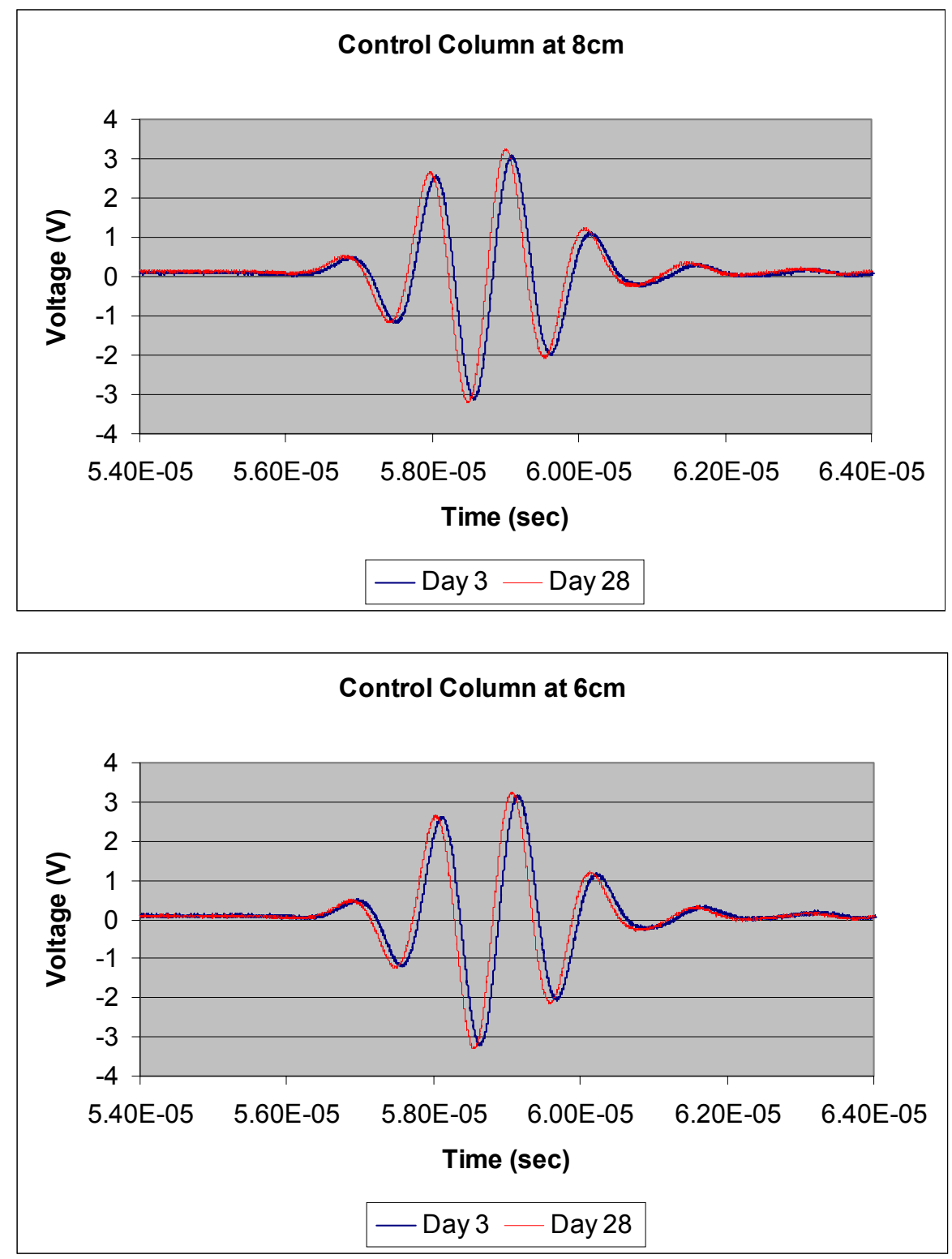

Figures 3.10 and 3.11 : A coustic w aveform data from the control colum n for the $8 \mathrm{~cm}$ and $6 \mathrm{~cm}$ depth locations over two periods of tim e. N ote the high degree of repeatability of the w aveform s. 
Two of the most interesting and variable features in the data are the waveform amplitudes and wave travel times, both of which vary as the experiment progresses. The amplitudes will be discussed further in the following section; however, the travel times bear mentioning here.

All of the measurement positions along the inoculated column exhibit decreases in travel time over the course of the experiment. The average column velocity (comprised of the sediment, fluid, and gas velocities), therefore is increasing over time. This is evident when one examines the time at which wave energies first arrive at the receiving transducer. For the periods measured, the decrease in travel time amounts to an average decrease of $0.6-1.0 \mu$ sec for the positions included here. For the most part, this decrease is very systematic. As time elapses during the experiment, the travel times are progressively shorter for each measurement period. The only exception appears to be for the $24 \mathrm{~cm}$ location between Days 11 and 28. Several explanations exist for the overall trend of decreasing travel times and these are detailed below.

The design of the tube within which the acoustic wave measurements were made (Figure 2.6) was such that the piezoelectric source and receiver transducers could not be permanently fixed at one location without the sediment column remaining in the fluid bath. As constant conditions were desired for each of the three measurement columns, it was decided that leaving the acoustic column in the fluid bath permanently might be 
undesirable due to temperature effects. Therefore, the acoustic column was removed from the measurement tube after each set of measurements was completed. This no doubt resulted in a slight error in the relocation of the acoustic source and receiver transducers. Such a relocation error could result in the transducers being either too close or too far away from one another with respect to the previous set of measurements. Any change in their separation from one period to the next would lead to a slight change in the wave travel time. While this may contribute to some error in measurement repeatability, it may not be the principle cause of the observed travel time decreases, however. Examination of the control column waveforms reveals no observable change in wave travel times over the course of the experiment. This likely implies that another mechanism is responsible.

An alternative explanation for this effect could be increased effective elastic moduli of the sediments within the column due to gradually increasing pressures as gas evolution occurs. Because the columns were maintained as closed systems, no fluid flow occurred through the sediments over the duration of the experiment (the exception being for those times during which $\mathrm{K}_{\mathrm{s}}$ measured). As dinitrogen was being produced and prevented from escape, the columns no doubt underwent some degree of pressurization. The wave velocity (and hence the wave travel time) is determined through the following relationship: 


$$
v=\sqrt{\frac{\mathrm{K}+4 / 3 \cdot \mu}{\rho}}
$$

where $v$ is the wave velocity, $K$ is the effective bulk modulus, $\mu$ is the effective shear modulus, and $\rho$ is the effective density (with all properties comprised of sediment, fluid, and gas contributions). The pressurization of the columns would likely lead to an increase in the effective elastic moduli, thus leading to an increase in velocity. Any increase in velocity would result in shorter travel times with the arrival of the acoustic wave occurring earlier in the time window. Similarly, the increase in column pressure and the concomitant increase in the elastic modulus may also explain the increase in amplitudes seen in the shallower regions of the column. This effect is described in more detail in Section 3.3.2.

As can be seen, the waveform characteristics change dramatically for the deeper locations $(16 \mathrm{~cm}, 8 \mathrm{~cm}$, and $4 \mathrm{~cm})$ over the course of the experiment. In particular, the change in character over the period from 3 days to 28 days post saturation may be used to examine in more detail the general changes observed in much of the data. The data are compared for these two periods for each of the four locations and are included as Figures 3.12 to 3.15 . Note that the voltage scales for the two data acquisition periods are now different so that the signals can be shown with approximately the same amplitude. 

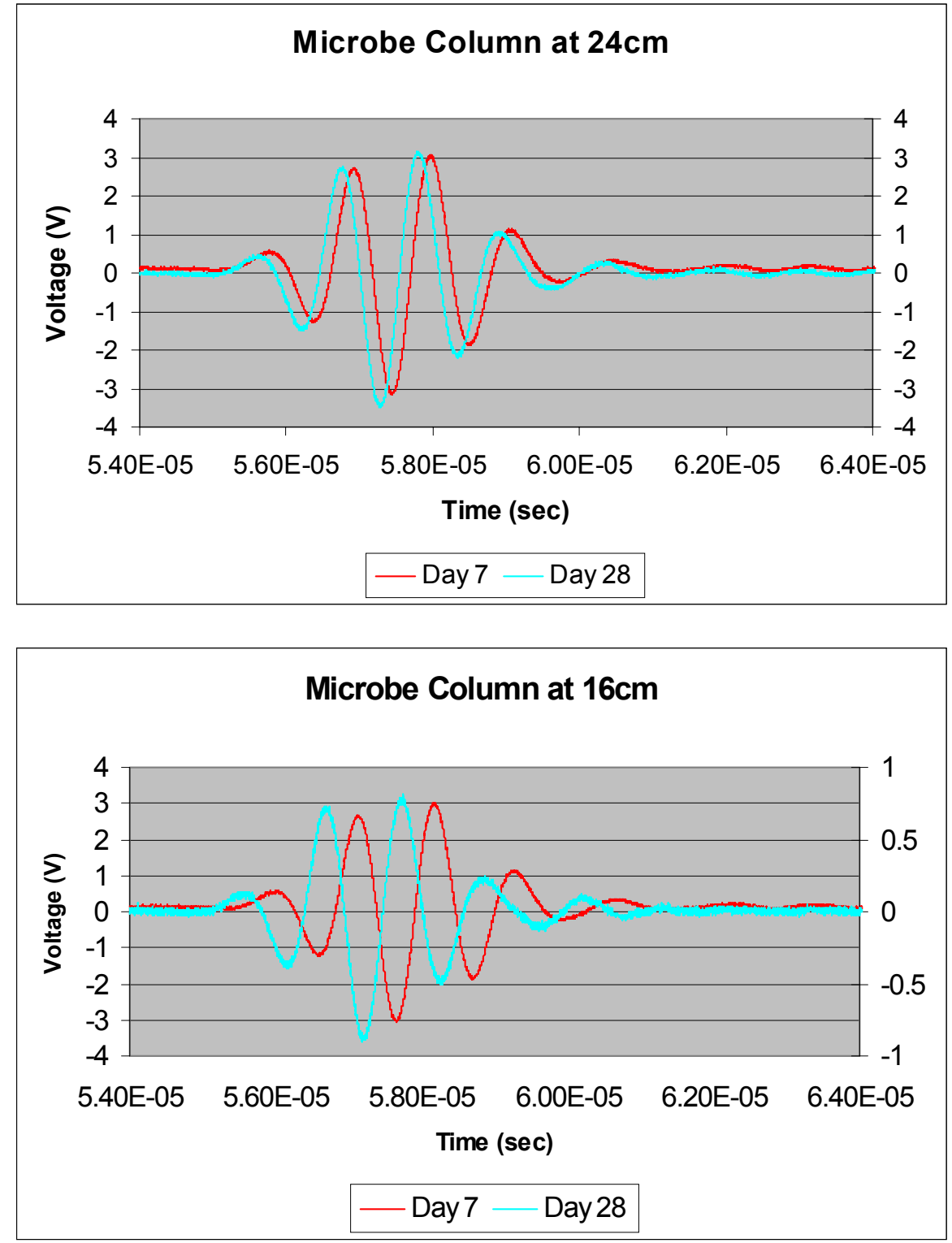

Figures 3.12 and 3.13: Acoustic waveform data from the inoculated colum $\mathrm{n}$ for the $24 \mathrm{~cm}$ and $16 \mathrm{~cm}$ depth locations over tw 0 periods of tim e. The voltage scale for the attenuated signal (D ay 28) is show $n$ on the right. $\mathrm{N}$ ote the differences in w ave travel tim $e$. 

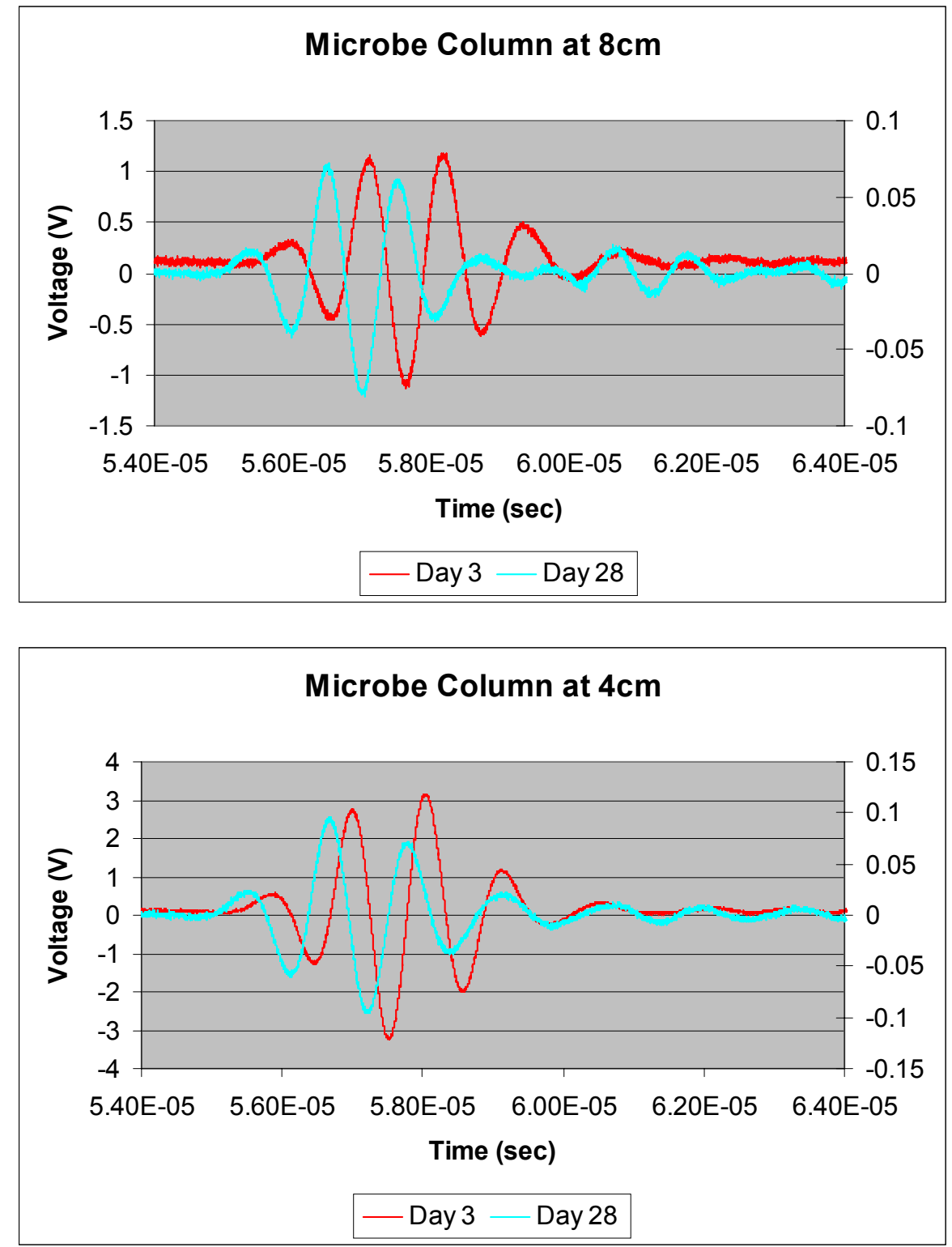

Figures 3.14 and 3.15: Acoustic waveform data from the inoculated colum $\mathrm{n}$ for the $8 \mathrm{~cm}$ and $4 \mathrm{~cm}$ depth locations over tw 0 periods of tim $\mathrm{e}$. The voltage scale for the attenuated signal (D ay 28) is shown on the right. $\mathrm{N}$ ote the differences in travel tim es. 
The compared waveforms show a variety of features that were not as clear in the earlier figures. Most striking is the difference between the waveform amplitudes for those locations deeper in the column as compared to the position just below the sediment water interface $(24 \mathrm{~cm})$. The data comparison for the $24 \mathrm{~cm}$ location shows that very little attenuation of the acoustic wave amplitude has occurred - even after a period of nearly a month during which time significant gas was evolved. This is most likely due to the low overburden pressure at this location relative to positions deeper within the column. Any gas bubbles formed near the interface were not easily inhibited from buoyant rise and loss to the headspace. Any gas evolved in the pore spaces around this depth was simply lost as the bubbles coalesced, overcame capillary pressures, and displaced sediment as they rose.

This phenomenon has been described as a common form of bubble transport through poorly confined sediment [Dullien, 1979, Anderson et. al., 1998] and was confirmed visually through the headspace window mentioned earlier. Small mounds first developed along the sediment-fluid interface followed by ebullition of the trapped gas. The idea that perhaps microbial biomass was simply too low in this region to promote significant gas generation was discounted due to the uniform distribution of microbes ultimately found in this section (see Section 3.4). 
Another feature of the waveform data worth mentioning is the trailing characteristics of the waveforms. In particular, the data compared for the $8 \mathrm{~cm}$ location shows an unusual feature. Late arriving low amplitude wave energy around 60-62 $\mu$ sec in the Day 28 record may represent scattered arrivals. One of the prime causes of acoustic wave attenuation is loss due to bubble scattering [Wilkins et. al., 1998]. For those bubbles with dimensions comparable to the wavelength of the transmitted acoustic wave, scattering of energy is a prime loss mechanism. Based on a presumed velocity of the saturated sediment of $1500 \mathrm{msec}^{-1}$ and a transmitting frequency of $1 \mathrm{MHz}$, a nominal wavelength of $1.5 \mathrm{~mm}$ is inferred. Given that accumulations of bubbles averaging $1-10 \mathrm{~mm}$ in diameter were being seen in the column by this time (28 days post saturation), it is likely that the some of the transmitted wave energy was being scattered by such bubbles. The waveform data may bear this out; however, it could also be simply the result of comparing features at greatly exaggerated scales. Further discussion of other attenuation mechanisms will be presented in the following section.

\subsubsection{Acoustic Wave Amplitude Data}

The amplitude for each of the measurement locations $(24-1 \mathrm{~cm})$ was determined through analysis of the waveform data and computed by measuring the maximum voltage from peak to trough. The amplitudes 
are included in Table 3.2. Also included for comparison are the data acquired for the control column for dates corresponding to 3 and 28 days post saturation. Both sets of data are presented in a series of images (Figure 3.16 and 3.17 ) which attempt to represent both the conditions and changes down the column over time.

Table 3.2: Amplitude Data

\begin{tabular}{|ccccccccc|}
\hline $\begin{array}{c}\text { Amplitude (V) } \\
\text { depth }(\mathrm{cm})\end{array}$ & $\begin{array}{c}\text { Control } \\
\text { Day 3 }\end{array}$ & Day 28 & $\begin{array}{c}\text { May 3 } \\
\text { Dacrobe }\end{array}$ & Day 4 & Day 7 & Day 10 & Day 11 & Day 28 \\
\hline \hline 24 & 5.650 & 5.639 & 5.819 & 6.316 & 6.219 & 6.112 & 5.686 & 6.660 \\
23 & 6.501 & 6.477 & 5.546 & 6.221 & 6.193 & 6.065 & 5.865 & 6.680 \\
22 & 6.510 & 6.562 & 5.415 & 5.989 & 6.147 & 5.931 & 5.754 & 2.600 \\
21 & 6.602 & 6.660 & 5.524 & 5.988 & 6.316 & 5.787 & 5.667 & 5.060 \\
20 & 6.660 & 6.647 & 5.506 & 5.836 & 6.243 & 5.822 & 5.552 & 6.500 \\
19 & 6.506 & 6.537 & 5.235 & 5.702 & 5.709 & 4.941 & 5.041 & 5.540 \\
18 & 6.410 & 6.432 & 5.445 & 5.636 & 6.019 & 1.957 & 0.913 & 2.420 \\
17 & 6.405 & 6.461 & 5.313 & 5.669 & 6.016 & 1.038 & 0.600 & 0.996 \\
16 & 6.607 & 6.627 & 5.294 & 5.588 & 6.081 & 1.651 & 1.311 & 1.710 \\
15 & 6.540 & 6.467 & 5.254 & 5.691 & 6.137 & 1.355 & 1.079 & 1.510 \\
14 & 6.502 & 6.585 & 5.414 & 5.497 & 6.140 & 1.975 & 1.904 & 2.130 \\
13 & 6.510 & 6.531 & 5.356 & 5.590 & 6.206 & 4.101 & 3.793 & 3.190 \\
12 & 6.403 & 6.480 & 5.110 & 5.281 & 6.079 & 1.272 & 1.124 & 1.280 \\
11 & 6.434 & 6.469 & 5.346 & 5.349 & 6.133 & 2.725 & 2.277 & 2.050 \\
10 & 6.561 & 6.550 & 5.014 & 5.272 & 6.070 & 1.937 & 1.448 & 0.324 \\
9 & 6.454 & 6.462 & 5.335 & 5.252 & 5.037 & 0.970 & 0.788 & 0.314 \\
8 & 6.475 & 6.479 & 4.804 & 4.586 & 2.311 & 0.450 & 0.751 & 0.153 \\
7 & 6.772 & 6.774 & 5.257 & 5.099 & 6.305 & 1.735 & 2.010 & 0.772 \\
6 & 6.502 & 6.564 & 4.925 & 4.715 & 5.360 & 0.698 & 0.307 & 0.280 \\
5 & 6.464 & 6.513 & 5.637 & 5.158 & 6.619 & 0.304 & 0.367 & 0.204 \\
4 & 6.402 & 6.444 & 5.505 & 5.283 & 6.410 & 0.581 & 0.437 & 0.192 \\
3 & 6.410 & 6.329 & 5.807 & 5.727 & 6.307 & 2.635 & 1.170 & 0.564 \\
2 & 5.701 & 5.830 & 5.481 & 5.498 & 6.016 & 3.852 & 1.677 & 0.422 \\
1 & 5.603 & 5.585 & 4.932 & 4.506 & 5.443 & 4.887 & 2.304 & 0.346 \\
\hline
\end{tabular}

The general trend of the amplitude data is unmistakable. The bulk of the microbe-inoculated column saw very little decrease in amplitude over the first week of the experiment. This was followed quite rapidly by a dramatic decrease in amplitudes as time goes on, undoubtedly a result of bubble accumulation within the sediment pore space. Ultimately, 

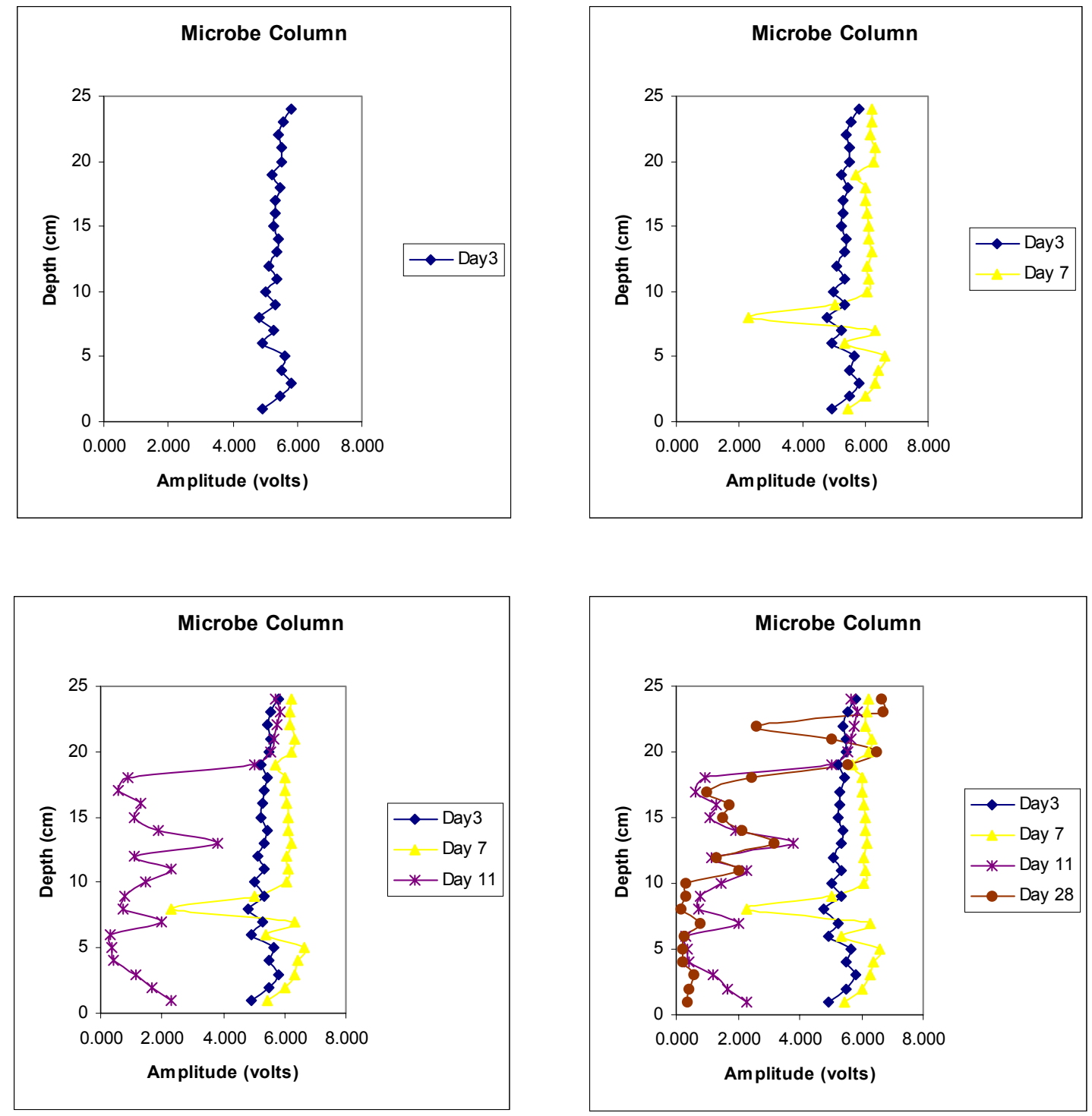

Figures 3.16: Series of graphs ilhustrating change in the m easured wave am plitudes over the four periods show $\mathrm{n}$ in Figures 3.4 to 3.7 . The onset of gas at the $8 \mathrm{~cm}$ location is follow ed by abrupt gas production throughout the colum $n$ shortly thereafter. $N$ ote the lack of attenuation of those $w$ aves transm itted through the upperm ost portion of the colum $n$. 

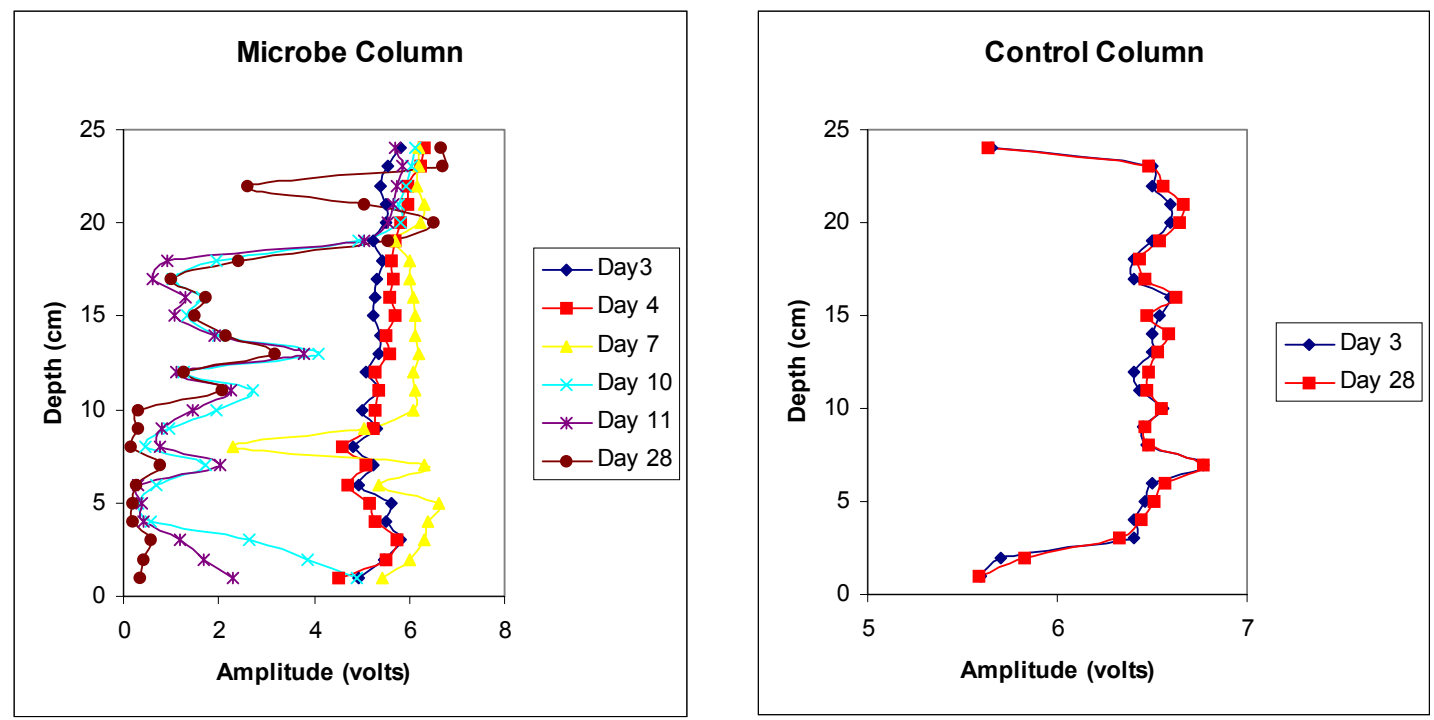

Figures 3.17: G raph of changing w ave am plitudes over the experim ent for both the $m$ icrobe-inoculated and the control colum $n$. N ote the low er values both high and low in the control colum $\mathrm{n}$ which are likely due to initially entrapped air during saturation. There is no observed tem poral change, how ever. 
amplitudes decrease over an order of magnitude throughout much of the column with the greatest decreases occurring deeper in the column.

Another interesting feature of the data is that the amplitudes appear to have increased throughout much of the column early on. Again, the effect of even a slight increase in the pressurization of the column due to some gas production would likely lead to an increase in the elastic modulus of the sediment. Such an increase might be expected to enhance wave propagation through increased grain contact stiffness, which would in turn increase the amplitudes. Wave energy loss due to bubble formation may not be occurring at this point due to the small size of the produced bubbles. Recall that upon bubble nucleation, bubbles may be entrained along the sediment surface before assimilating into pore filling gas pockets [Ronen et. al., 1989]. The frequency of the transmitted waves $(1 \mathrm{MHz})$ is still low enough and the wavelength long enough $(1.5 \mathrm{~mm})$ that the impact of very small bubbles $(<10 \mu$ m diameter) may be imperceptible. By combining these two effects, the initial observed amplitude increase might be a reasonable expectation of the proposed bubble model.

\subsubsection{Acoustic Wave Attenuation Coefficients}

In an attempt to better compare the results of the amplitude decreases with those of similar published results [Wilkins et al., 1998], they were quantified in terms of the attenuation coefficients for each position over 
time. This was done by using the following relationship detailed in Johnston and Toksöz [1981]:

$$
\alpha=8.686\left(\frac{-\ln \left(\mathrm{A} / \mathrm{A}_{\mathrm{O}}\right)}{\mathrm{x}}\right)
$$

where $\alpha$ is the attenuation coefficient $\left[\mathrm{dBm}^{-1}\right], \mathrm{A}$ is the observed amplitude in volts, $A_{0}$ is the initial amplitude in volts, and $\mathrm{x}$ is the distance the wave travels through the column in meters.

The measured amplitudes used to determine $\alpha$ are those listed in Table 3.2 with the initial amplitude taken as that recorded 3 days after column saturation (i.e. the first day on which data were recorded). The results are listed in Table 3.3, and they agree nicely with those reported by Wilkins et al. [1998]. In that study, high frequency waves $(38-400 \mathrm{kHz})$ were used to measure attenuation coefficients in gassy sediments from diver-retrieved cores from Eckernförde Bay. The values reported for $\alpha$ range from 50-500 $\mathrm{dBm}^{-1}$ depending upon the measurement frequency and sample condition. Although the sediment type and wave frequency differ somewhat from those used here, it is clear that the results reported in Table 3.3 agree with other published data. 
Table 3.3: Attenuation coefficients for the column over time

\begin{tabular}{|ccccc|}
\hline $\begin{array}{c}\text { Attenuation } \\
\text { Days 3-4 }\end{array}$ & $\begin{array}{c}(\mathrm{dB} / \mathrm{m}) \\
\text { Days 3-7 }\end{array}$ & $\begin{array}{c}\text { Days 3-10 } \\
\text { Days 3-11 }\end{array}$ & Days 3-28 \\
\hline \hline-11.81 & -9.58 & -7.08 & 3.33 & -19.44 \\
-16.54 & -15.89 & -12.89 & -8.06 & -26.80 \\
-14.51 & -18.26 & -13.11 & -8.75 & 105.68 \\
-11.62 & -19.30 & -6.70 & -3.68 & 12.64 \\
-8.38 & -18.10 & -8.04 & -1.20 & -23.91 \\
-12.31 & -12.49 & 8.33 & 5.44 & -8.16 \\
-4.97 & -14.44 & 147.40 & 257.23 & 116.81 \\
-9.34 & -17.90 & 235.21 & 314.16 & 241.16 \\
-7.79 & -19.96 & 167.84 & 201.06 & 162.78 \\
-11.51 & -22.38 & 195.21 & 228.02 & 179.61 \\
-2.19 & -18.13 & 145.26 & 150.53 & 134.38 \\
-6.16 & -21.22 & 38.46 & 49.70 & 74.64 \\
-4.74 & -25.01 & 200.31 & 218.13 & 199.41 \\
-0.08 & -19.78 & 97.07 & 122.94 & 138.07 \\
-7.23 & -27.53 & 137.00 & 178.91 & 394.58 \\
2.26 & 8.28 & 245.56 & 275.50 & 408.03 \\
6.69 & 105.41 & 341.10 & 267.32 & 496.49 \\
4.40 & -26.19 & 159.68 & 138.49 & 276.33 \\
6.28 & -12.19 & 281.45 & 399.76 & 413.02 \\
12.79 & -23.13 & 420.63 & 393.50 & 478.09 \\
5.93 & -21.92 & 323.91 & 364.94 & 483.41 \\
2.00 & -11.90 & 113.82 & 230.77 & 335.88 \\
-0.45 & -13.42 & 50.80 & 170.59 & 369.34 \\
13.01 & -14.20 & 1.32 & 109.63 & 382.74 \\
\hline
\end{tabular}

Of note are the negative values for $\alpha$ (i.e. increased amplitudes over time) for those positions near the top of the column. An explanation for this observation has already been provided in Section 3.3.3, but it is worth noting again, as an average value for $\alpha$ may be determined from this region and used as a pressure correction for the deeper values. This value amounts to an average decrease in attenuation (i.e. increase in amplitude) of $-23.12 \mathrm{dBm}^{-1}$ over the duration of the experiment. Using both this value and the amplitude of an acoustic wave propagated through a column containing only water (i.e. no sediment), the attenuation due solely to the 
saturated sediments and the attenuation due to the gassy sediments may be determined. From this, the attenuation due exclusively to gas-filled pore spaces may be determined. The values are included in Table 3.4 with the amplitude for the wave propagated through the water-filled column being 14.30 volts. For comparison, the amplitude and travel time differences for the two scenarios (sediment and water) are included as coplotted waveforms in Figure 3.18.

Table 3.4: Attenuation of saturated vs. gassy sediments

\begin{tabular}{|ccc|}
\hline $\begin{array}{c}\text { Attenuation due to } \\
\text { sat sed's }(\mathrm{dB} / \mathrm{m})\end{array}$ & $\begin{array}{c}\text { Max attenuation due to } \\
\text { gassy sed's }(\mathrm{dB} / \mathrm{m})\end{array}$ & $\begin{array}{c}\text { Attentuation due to } \\
\text { gas only }(\mathrm{dB} / \mathrm{m})\end{array}$ \\
\hline \hline 129.49 & 110.04 & -19.44 \\
136.41 & 109.61 & -26.80 \\
139.85 & 245.53 & 105.68 \\
136.98 & 149.62 & 12.64 \\
137.45 & 113.54 & -23.91 \\
144.72 & 136.56 & -8.16 \\
139.06 & 255.87 & 116.81 \\
142.59 & 383.75 & 241.16 \\
143.11 & 305.89 & 162.78 \\
144.20 & 323.81 & 179.61 \\
139.88 & 274.25 & 134.38 \\
141.43 & 216.07 & 74.64 \\
148.20 & 347.61 & 199.41 \\
141.70 & 279.77 & 138.07 \\
150.93 & 545.51 & 394.58 \\
142.00 & 550.03 & 408.03 \\
157.10 & 653.59 & 496.49 \\
144.12 & 420.45 & 276.33 \\
153.51 & 566.54 & 413.02 \\
134.06 & 612.15 & 478.09 \\
137.48 & 620.88 & 483.41 \\
129.78 & 465.67 & 335.88 \\
138.11 & 507.45 & 369.34 \\
153.31 & 536.05 & 382.74 \\
\hline
\end{tabular}




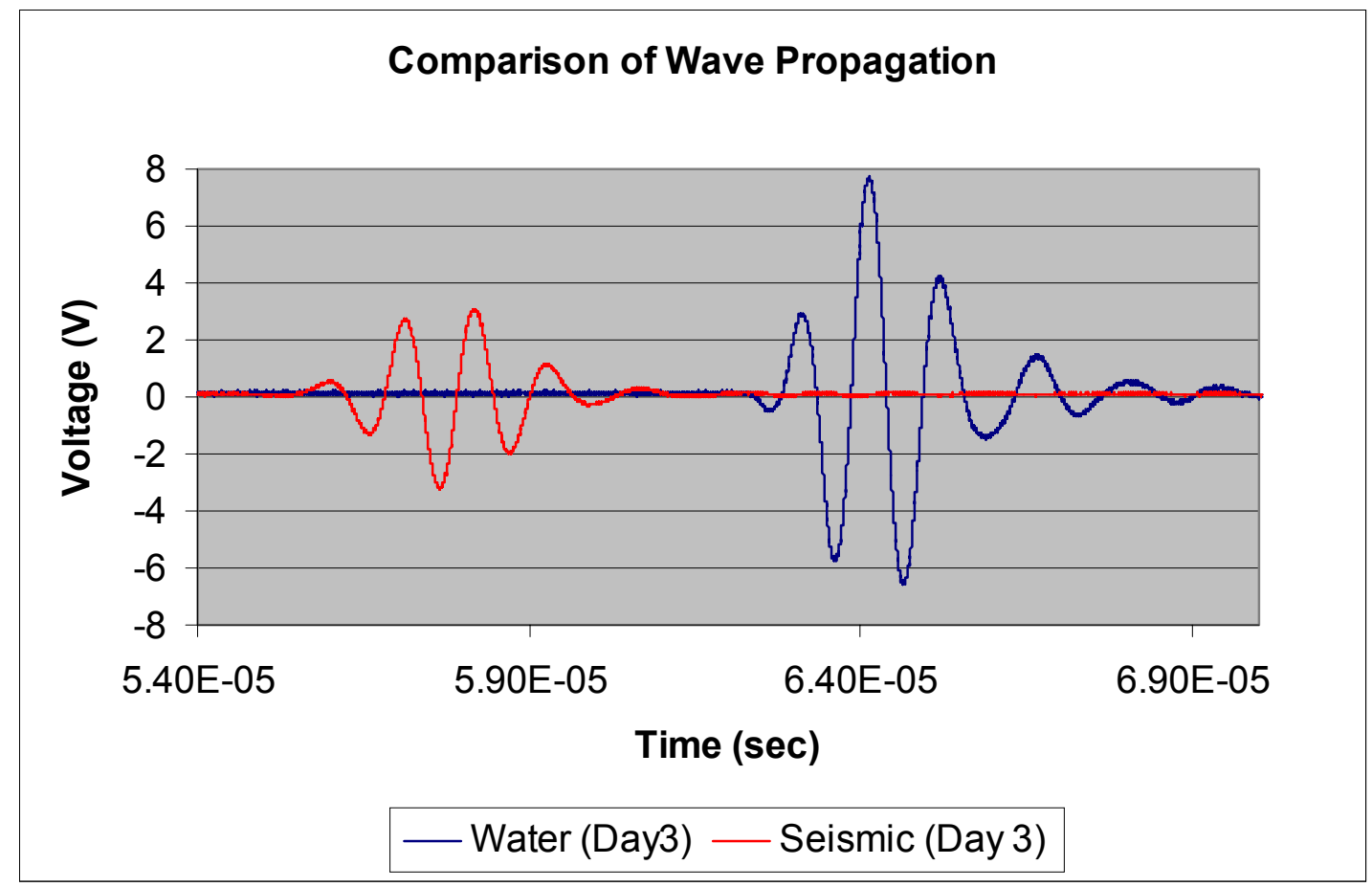

Figures 3.18: A coustic w aveform data ilhustrating the difference in wave am plitude and travel tim e for a wave transm itted through a sedim entfilled and a water-filled colum $\mathrm{n}$. The am plitude from the latter w as used to determ ine the attenuation coefficient of the sedim ent alone (i.e. no gas). 
It is worth recalling here that the sediments themselves act to dissipate the energy of a transmitted acoustic wave, particularly at the high frequencies being used in this experiment. The point of calculating the effect of the gas bubbles alone then was to get a handle on the degree of attenuation occurring and to speculate on the primary mechanism of wave attenuation. This shall be discussed further in Chapter 4 where some preliminary modeling of the expected bubble resonance frequencies is presented.

\subsubsection{Acoustic Wave Spectra}

It is also appropriate to include the spectral data that resulted from analysis of the acoustic waveforms. The spectral analysis was accomplished using the Tektronix TDS-3012 oscilloscope and the built-in Fast Fourier Transform (FFT) function. The spectral results may be used to assess the degree to which certain frequencies within the relatively narrow bandwidth of the acoustic wave pulse were attenuated. Two locations down the length of the microbe column $(16 \mathrm{~cm}$ and $8 \mathrm{~cm})$ as well as one from the control column $(12 \mathrm{~cm})$ were chosen for spectral waveform analysis with the results included as Figures 3.19 to 3.21 . It should be noted that the vertical scale consists of the portion of the waveform amplitude comprised of a given frequency and that the scales have been adjusted where appropriate for better spectral comparison. 


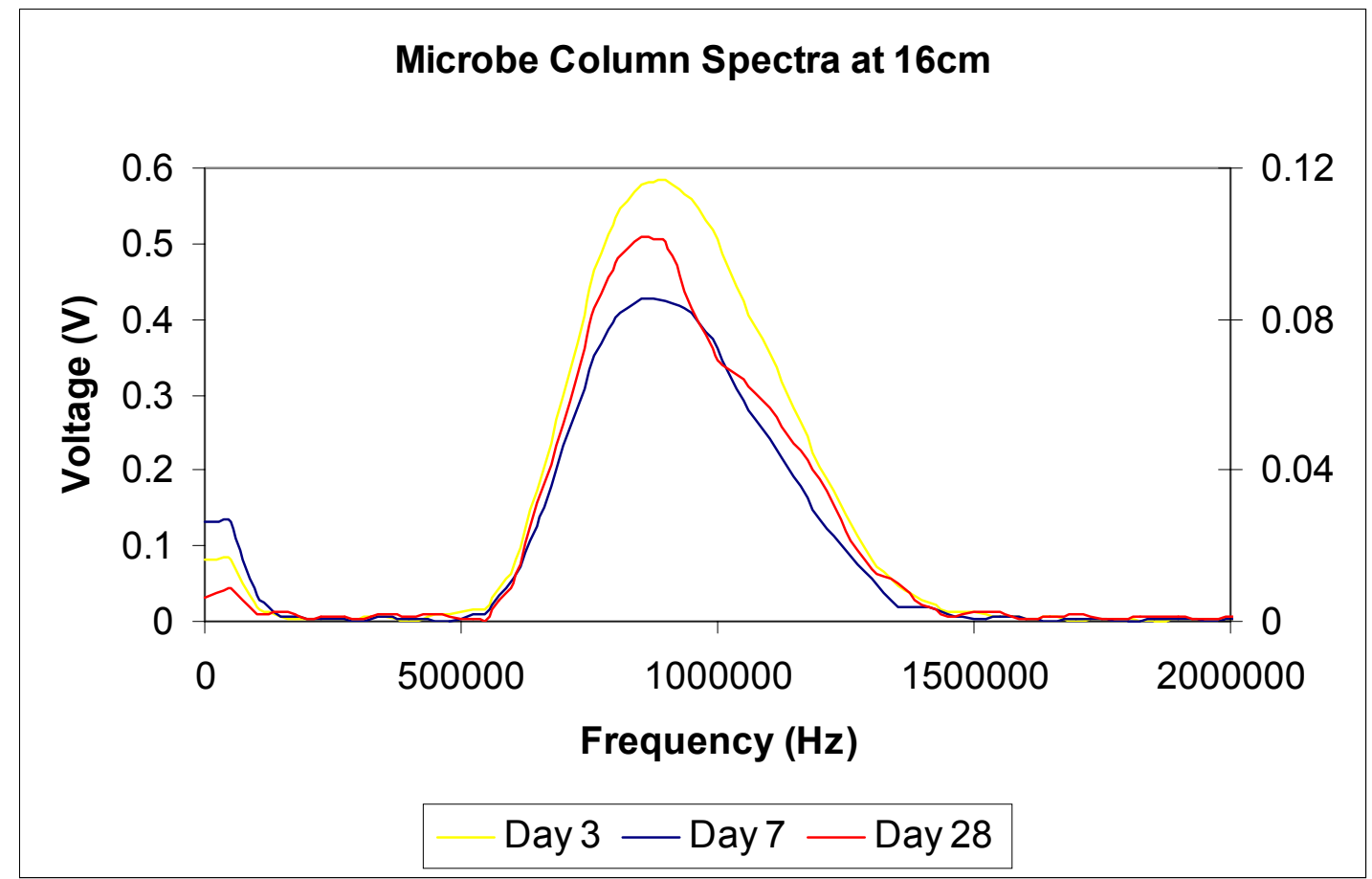

Figures 3.19: Inoculated colum n w aveform spectra for the $16 \mathrm{~cm}$ location over three tim e periods (D ay 28 voltage scale is on the right). N ote the selective attenuation of frequencies above $950 \mathrm{kH} \mathrm{z}$. These correspond to dam ping losses associated $w$ ith very sm all diam eter bubbles or $m$ ore likely, scattering off of larger diam eter bubbles. 


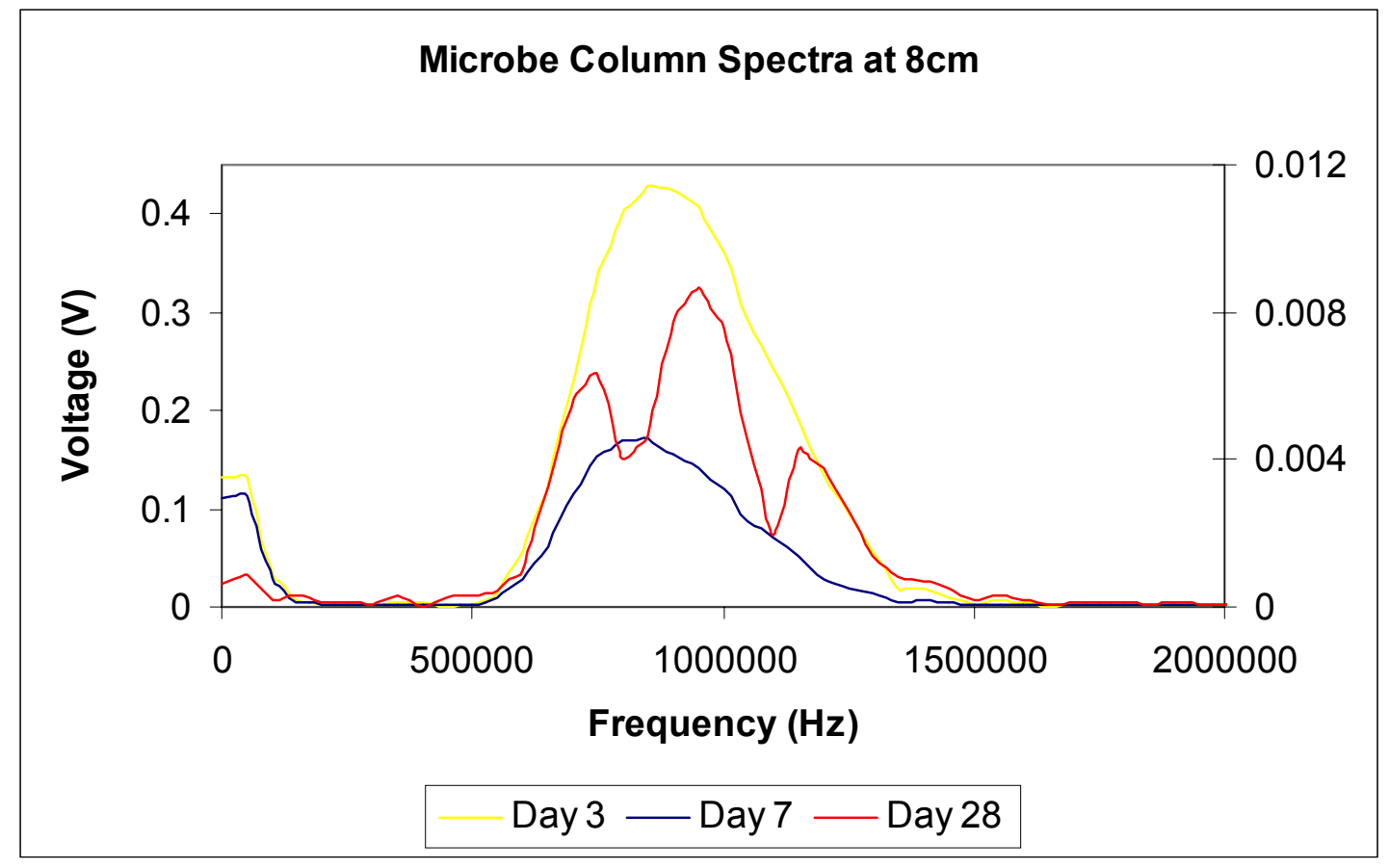

Figures 320 : Inoculated colum n waveform spectra for the $8 \mathrm{~cm}$ location over three tim e periods (D ay 28 voltage scale is on the right). N ote the selective attenuation of frequencies betw een 750 and $950 \mathrm{kH} \mathrm{z}$ and above $1 \mathrm{M} \mathrm{H} z$. These $\mathrm{m}$ ay correspond to both dam ping losses associated $\mathrm{w}$ ith a range of pore-filling bubble sizes and to w ave scattering. 


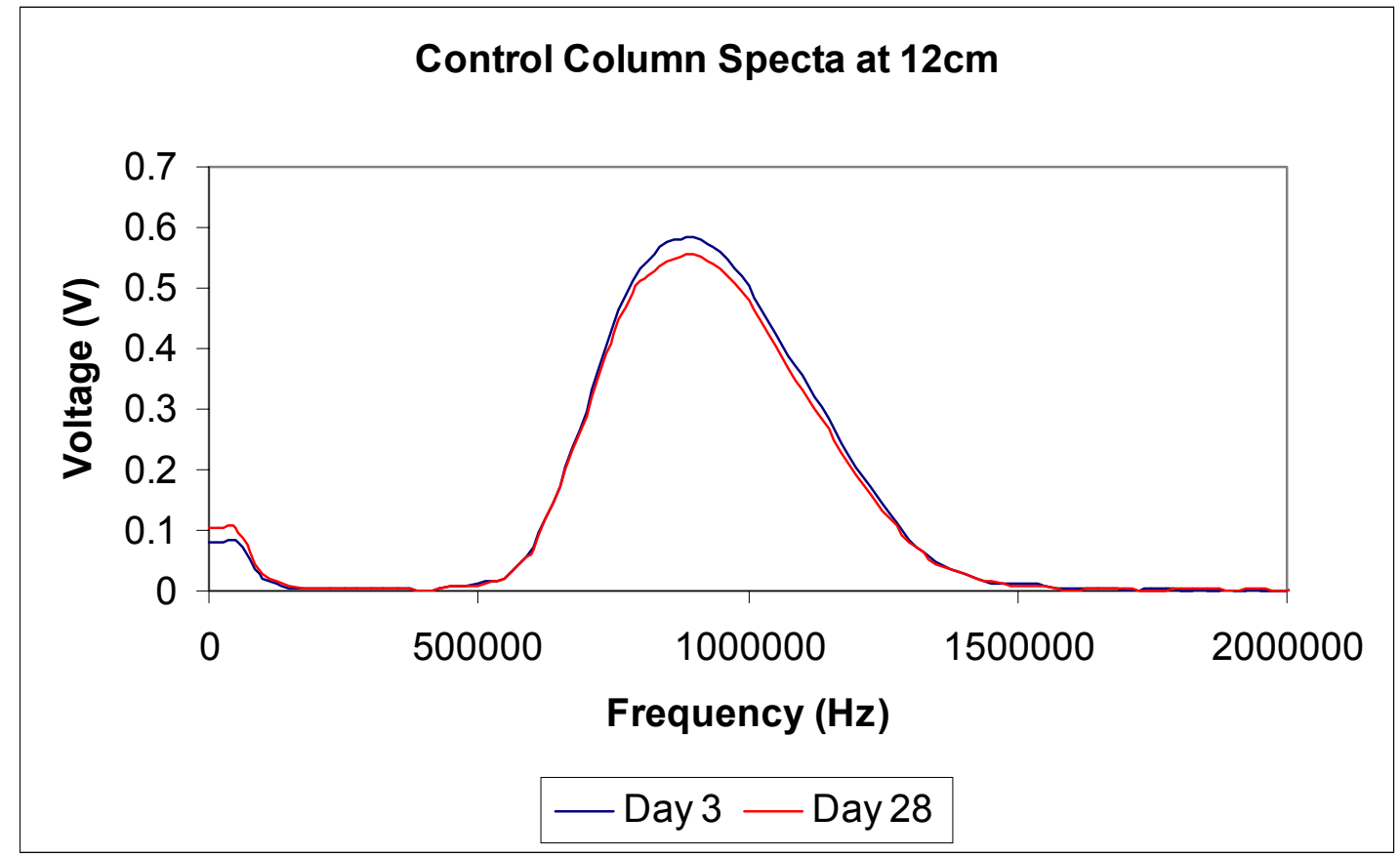

Figures 321 : Control colum n w aveform spectra for the $12 \mathrm{~cm}$ location over tw o tim e periods. N ote the high degree of repeatability in the tw o spectra over the course of nearly one $m$ onth. 
The spectral results clearly show the degree to which certain frequencies are attenuated over the course of the experiment. Namely, the highest frequencies within the effective bandwidth of the transducers $(\sim 750 \mathrm{kHz}$ to $1.25 \mathrm{MHz})$ are progressively attenuated as pore space gas filling occurs. Of particular note is the spectral character of the final waveform collected at the $8 \mathrm{~cm}$ position. The spectral results suggest what appears to be band pass filtering by the trapped gas bubbles. As shall be discussed further in Chapter 4, it appears from the data that as bubbles nucleate, become trapped, and grow in size, their attenuating properties change according. Variability in bubble size might be expected throughout the column with larger bubbles accumulating in sedimentdisplaced regions closer to the fluid interface where overburden pressures are lessened. That this is so may be inferred by comparing the results from the two locations (16 and $8 \mathrm{~cm}$ ) and their spectral change over time. Such an effect is commiserate with the expectations presented by the bubble models of Anderson et al. [1998] in which a variety of bubble accumulations and distributions within shallow ocean sediments are discussed.

\subsection{Microbial Biomass Estimation - Denitrification Assay}

The most probable number (MPN) of denitrifying bacteria per gram wet weight ranged from $\log 6.4$ to 9.0, with most values ranging between 
$\log 7.0$ to 8.4 (Table 3.5). In the one section tested for morphology of the colonies on solid medium, greater than $98 \%$ of the colony forming units had the distinctive colony morphology of Pseudomonas stutzeri KC, indicating that little to no contamination by other denitrifying bacteria occurred during the experiment. The mean population of cells in the sand samples was $1.51 \times 10^{8}(\log 8.18)$ cells per gram wet weight. This represents at least one order of magnitude of growth in the column, and is $70 \%$ of the expected growth based on the full utilization of acetate and nitrate in the column and the assumed stoichiometric conversion to biomass.

It is possible that only $20 \%$ or less of the bacteria added to the sand remained after the sand was loaded into the column and subjected to flow. Alternatively, the stoichiometric coefficients for the dissimilatory versus assimilatory half reactions may have been slightly incorrect. This seems like a reasonable possibility given that a larger percentage of the available nitrate may have been used for energy generation as opposed to cell synthesis. Given a modest change in the dissimilatory half reaction fraction from $80 \%$ to $88 \%$, the resulting biomass prediction $\left(4 \times 10^{8}\right.$ cells $/ \mathrm{g}$ sediment) agrees very closely with that measured using the MPN analysis (1.51 x $10^{8}$ cells/g sediment). 
Table 3.5: MPN of denitrifying bacteria per gram wet weight

\begin{tabular}{|cccc|}
\hline depth $(\mathrm{cm})$ & MPN & denitrifying activity, log(MPN) & \\
\hline \hline 28.00 & $9.33 \mathrm{E}+07$ & 7.97 & free water at top of sand \\
25.50 & $4.27 \mathrm{E}+07$ & 7.63 & top of sand column \\
22.96 & $2.40 \mathrm{E}+07$ & 7.38 & \\
20.42 & $2.40 \mathrm{E}+08$ & 8.38 & \\
17.88 & $4.27 \mathrm{E}+07$ & 7.63 & \\
15.34 & $2.40 \mathrm{E}+07$ & 7.38 & \\
12.80 & $9.33 \mathrm{E}+08$ & 8.97 & \\
10.26 & $9.33 \mathrm{E}+07$ & 7.97 & \\
7.72 & $2.40 \mathrm{E}+08$ & 8.38 & \\
5.18 & $2.31 \mathrm{E}+06$ & 6.36 & bottom of sand column \\
2.64 & $9.33 \mathrm{E}+06$ & 6.97 & \\
0.10 & $9.33 \mathrm{E}+06$ & 6.97 & \\
\hline
\end{tabular}




\section{Chapter 4}

\section{Discussion and Conclusions}

\subsection{Introduction}

In this study, a variety of methods were used to characterize the impact of microbe generated gas on column studies under conditions favorable to denitrification. The methods were chosen so as to ascertain the origin of the evolved gas (e.g. biotic or abiotic), to confirm its composition and distribution, and to gauge its impact. Central to this study was the use of a geophysical technique, acoustic wave propagation, designed to extend the reach and resolution of the more widely utilized methodologies.

The results may be taken together to establish a general picture for the conditions evolving within the column over the duration of the 
experiment. Analysis of the acoustic waveform data along with some preliminary modeling, however, allows for a much more specific and detailed picture to be developed of the prime factor restricting column flow: microbial-respired bubbles.

\subsection{Assessment of Temporal Column Conditions}

Measurement of the saturated hydraulic conductivity indicates that the conditions within the column are such that pore throats and networks are being affected in such a way as to restrict the flow of fluid. The data suggest that the effect occurs slowly at first but that the rate of decrease rapidly increases over a very short time. Similarly, the mass spectrometer analysis of the sampled pore spaces suggests that some fraction of the pore spaces is filling with ${ }^{15} \mathrm{~N}$-enriched dinitrogen during this same period. As a similar effect is not observed in the non-inoculated control column, the only likely source for such gas would be through the respiratory activity of the denitrifying microbes resident within the column. That these events are similarly located in time suggests that the largest effects may be arising during the exponential phase of microbial growth.

In contrast, the time period immediately following column saturation was not characterized by the any of the anticipated effects. Rather, it appears that the microbes were acclimating themselves with the 
newfound conditions of fluid saturation, mild anaerobiosis, and a change in substrate supply and type. Recall that the microbes were initially grown to their starting concentration aerobically on a complex TSB medium and that they were added to the columns adhered to relatively desiccated sediment. Upon wetting with the de-aired defined growth medium there was very likely a period of cell lysis followed by enzyme synthesis (e.g. nitrate reductase) commiserate with growth under denitrifying conditions. It seems likely that this period would be characterized by little to no growth or respiratory activity, at least none significant enough to induce observable changes. The data appear to bear out this inference.

\subsection{Acoustic Estimation of Bubble Development}

As mentioned, the acoustic technique may be used to directly probe the column for regions of bubble development and thus for regions of microbial growth and respiration. It may also be used to assess the type and degree of pore throat blocking due to bubble formation. As described in Anderson et al. [1998], there are several working theories governing the formation and entrapment of biogenic gas bubbles within saturated sediments.

The basic premise is that those gases produced through microbial catabolism in sufficient excess to exceed the solubility of the gas in a fluid 
may become trapped within the restrictive pore network of the hosting sediments. As the gas phase partitions within the pore space, bubble nucleation is expected to occur along the fixed surfaces of the grain boundaries or on colloids present in suspension [Dullien, 1979]. If the nucleated bubble remains adhered to such a surface (a very real possibility given the high surface tension of small bubbles) before being lost to buoyant rise, it becomes the growth point or receptacle for further gas phase partitioning. In this way, the bubble grows within the pore space until it is too large to be pushed through the pore throat under buoyant forces and thus becomes trapped. Bubble entrapment may be a permanent effect or it may be ephemeral. Given sufficient bubble connectivity within a pore network, the capillary force of the bubble mass may exceed that necessary for entrapment. At this point, the bubbles may rise through the sediment column through the deformation and displacement of the overlying sediments or through the deformation of the bubbles themselves.

The acoustic waveform data presented above may contain enough information to extract a picture of such bubble development in the column. By considering the potential range of bubble sizes within the column, we can analyze the attenuation and spectral data presented in Chapter 3 and compare these with the results of similar experiments. 
Before this can be done, however, the methods used to estimate potential bubble sizes must be discussed. They will be considered in terms of so-called pore volume. This term refers to the volume of an individual pore as represented by a perfect sphere and does not refer to the classic hydrologic definition of the term. There are several ways to estimate the maximum pore volume, and hence the largest bubble capable of filling such a pore. They are as follows:

1. Assume cubic packing of the sediment grains within the column. For a uniform grain size with a radius of $\sim 125 \mu \mathrm{m}$ (the case here), a maximum pore volume with a diameter of $340 \mu \mathrm{m}$ is the result [Collins, 1975].

2. Assess the size through the application of the mean hydraulic diameter: $\mathrm{D}_{\mathrm{h}}=4(\mathrm{~V} / \mathrm{S})$ where V/S is the volume to surface ratio of the pores. For grains with a circular cross section, $\mathrm{V} / \mathrm{S}=\mathrm{r} / 2$, and again assuming a grain size with a radius of $125 \mu \mathrm{m}, \mathrm{D}_{\mathrm{h}}=250 \mu \mathrm{m}$ for a bubble diameter of $250 \mu \mathrm{m}$ [Dullien, 1979].

3. Assess the size by using the microbial colony size/bubble radius relationship. This relationship doesn't account for pore size but rather estimates the size of a bubble derived from a microbial colony just before detachment occurs. This is the size the bubble reaches at the moment the buoyant force equals the surface tension holding the bubble to the colony and is equal to $R_{b}=233 R_{m}{ }^{0.3}$ where 
$R_{m}$ is the size of the microbe colony. Assuming an average colony size of $10 \mu \mathrm{m}$, the resulting bubble diameter is $\sim 700 \mu \mathrm{m}$ [Blanchard and Syzdek, 1977].

The above three methods yield a reasonable range of bubble diameters from $250-340 \mu \mathrm{m}$ with the potential for unusually large pores to accommodate even larger bubbles $(>600 \mu \mathrm{m})$. Further, networks of bubbles that bridge one or more pores would result in larger effective bubble sizes. It is this range of sizes that will be used to predict the effect of the bubbles on a range of acoustic wave frequencies.

The mechanisms by which bubbles act to attenuate acoustic wave energies is multi-fold and nicely described for the case of gassy sediments in Lyons et al. [1996], Anderson et al. [1980a, 1998], and Wilkins et al. [1998]. The primary mechanisms are through the scattering from bubbles and the internal absorption due to bubble wall motion (damping losses). While scattering will come into play for bubbles with dimensions comparable to the wavelength of the transmitted waves (Section 3.3.1), smaller bubbles should equally impact the wave amplitudes. The damping losses are a potentially very large source of energy attenuation, especially for very small bubble sizes. They arise as a result of energy transfer from the transmitted wave to the bubble and reach a peak at a particular resonance frequency. The resonance frequency of a given bubble is dependent upon - among other things - the bubble radius. As 
such, the utility of defining the resonance frequencies for a variety of bubble sizes in our denitrifying sediment columns will be examined.

Using a method similar to that of Anderson and Hampton [1980b], the resonance frequency $\left(f_{0}\right)$ for a given bubble radius may determined by way of the following expression:

$$
\mathrm{f}_{\mathrm{O}}=\frac{\left(\frac{3 \cdot \gamma \cdot \mathrm{P}_{\mathrm{O}}}{\mathrm{A} \cdot \rho}+\frac{4 \cdot \mathrm{G}}{\rho}\right)^{0.5}}{2 \cdot \pi \cdot \mathrm{r}}
$$

where the values used for the above expression are appropriate for the column conditions being studied here and are included in Table 4.1.

Table 4.1: Parameters used in resonance frequency calculation

\begin{tabular}{|ll|}
\hline \multicolumn{1}{|c|}{ Parameter Value } & \multicolumn{1}{c|}{ Parameter Definition } \\
\hline \hline$f_{o}$ & Resonance frequency $(\mathrm{Hz})$ \\
$\gamma=1.40$ & Ratio of the specific heats of the gas (nitrogen) \\
$P_{o}=1.013 \times 10^{6}$ & Ambient hydrostatic Pressure (dyne $/ \mathrm{cm}^{2}$ atm) \\
$G=4.5 \times 10^{9}$ & Dynamic shear modulus $\left(\right.$ dyne $\left./ \mathrm{cm}^{2}\right)$ \\
$A=1$ & Gas polytropic coefficient \\
$\rho=1.9$ & Bulk density of the sediment $\left(\mathrm{g} / \mathrm{cm}^{3}\right)$ \\
$r$ & Bubble radius $(\mathrm{cm})$ \\
\hline
\end{tabular}


A value for the bubble radius was not explicitly specified in the determination of $\mathrm{f}_{\mathrm{o}}$; rather a range of radii values from $10 \mu \mathrm{m}$ to $10,000 \mu \mathrm{m}$ was used. The resulting resonance frequencies are plotted as a function of radius and are included in Figure 4.1.

When the results are compared for the range of expected bubble sizes, it is clear that the calculated resonance frequencies lie within the bandwidth of frequencies used here. This observation helps to explain some of the extreme attenuation observed early on in the experiment and the resulting waveform spectra. In particular, the lower column positions (e.g. $8 \mathrm{~cm}$ ) and the bubbles developing there exhibit just the effect one would associate with attenuation due to resonance frequency of bubbles.

The earliest periods of respiration would no doubt result in nucleated bubbles whose dimensions are small enough that they would have resonance frequencies above the range of the system used here (750$1250 \mathrm{kHz}$ ). In instances where this is the case, attenuation of the waveform would be dominated by losses due to simple frame shearing within the sediment matrix. Based on other reported data, these losses are closely matched by those reported in Table 3.4 [Wilkins et al., 1998].

Once bubble aggregation occurs such that either the effective or true radius of a bubble results in a resonance frequency matching that of the transmitted wave, attenuation due to bubble damping will become a major factor. This is exactly what is observed at the $8 \mathrm{~cm}$ location in the microbe 

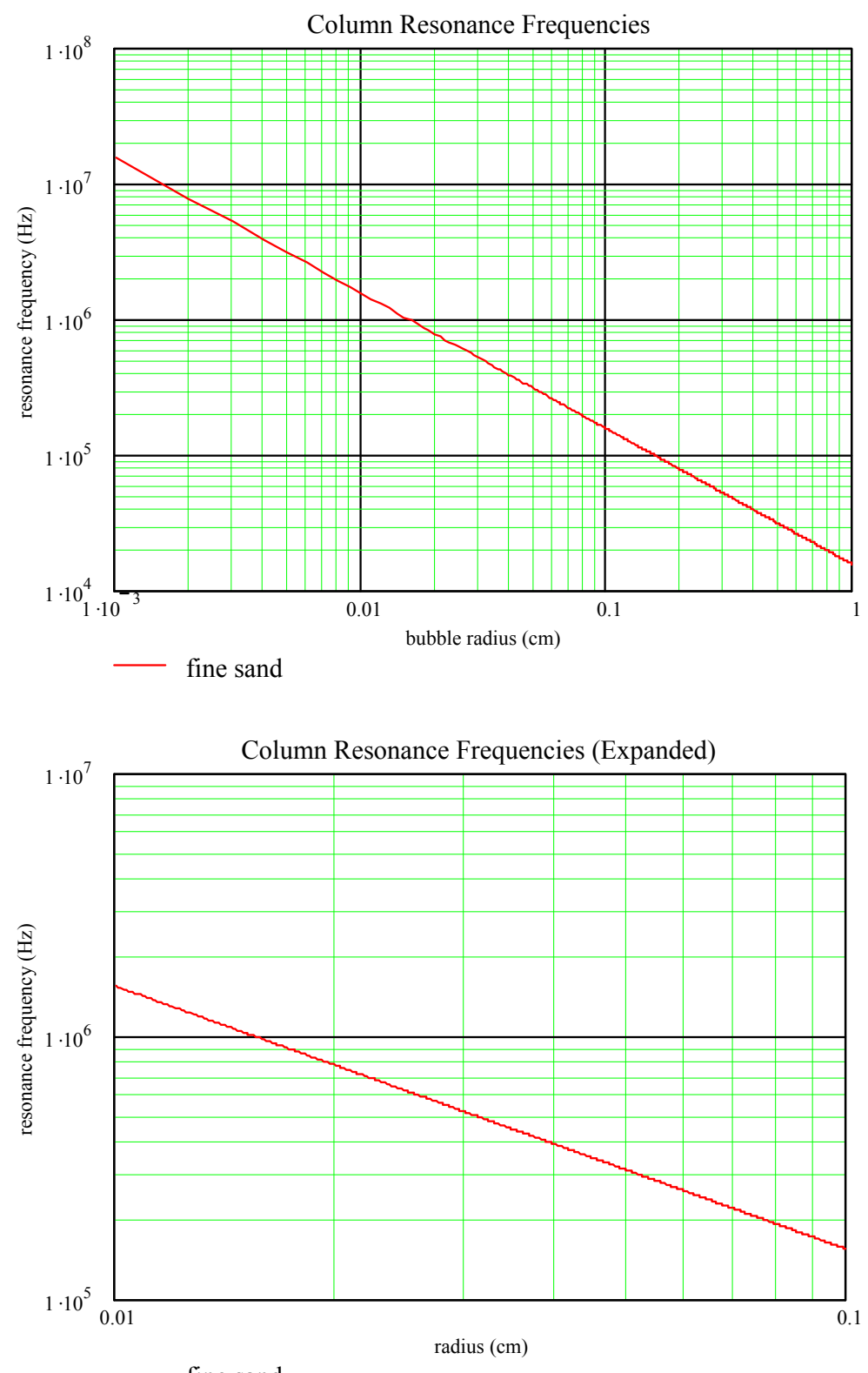

Figures 4.1: M athem atical estim ation of the resonance frequencies corresponding to a w ide range of bubble sizes (low er im age is an expanded view ). The param eters used here $m$ atch those believed to exist $w$ ith in the colum ns. 
column. What is more, the degree of attenuation observed in the microbe column $\left(200<\alpha<400 \mathrm{dBm}^{-1}\right)$ and listed in Table 3.4 closely matches that predicted and observed by other researchers investigating a similar effect [Wilkins et al., 1998].

This is made more apparent by comparing the modeling results (Figure 4.2) of an experiment utilizing a similar approach in which the impact of attenuation due to bubble damping is evident [Tuffin et al., 2001]. Here attenuation coefficients of $200-500 \mathrm{dBm}^{-1}$ are predicted. When one compares the spectral data from the $8 \mathrm{~cm}$ location (Figure 3.20), it is clear that the higher frequencies corresponding to the resonant bandwidth are being more severely attenuated than are others. Looking more closely at the results plotted in Figure 4.1, one finds that the resonance frequency range from $700-950 \mathrm{kHz}$ corresponds to bubble sizes ranging from $310^{-}$ $440 \mu \mathrm{m}$ in diameter. Recall that the bubble size estimations made above

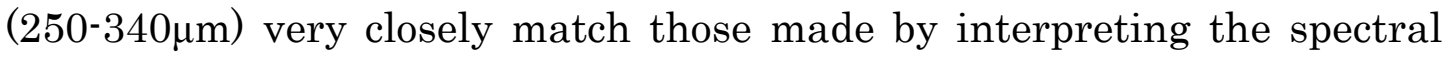
results. This is significant in that it offers up an alternative for characterizing bubble dimensions as well as spatial location within a column. Similar features of specific frequency attenuation are also observed for the $16 \mathrm{~cm}$ location, although not to the same degree. It is possible that the bubble sizes eight centimeters higher in the column were larger and hence fell out of the range of frequency used in the experiment. 


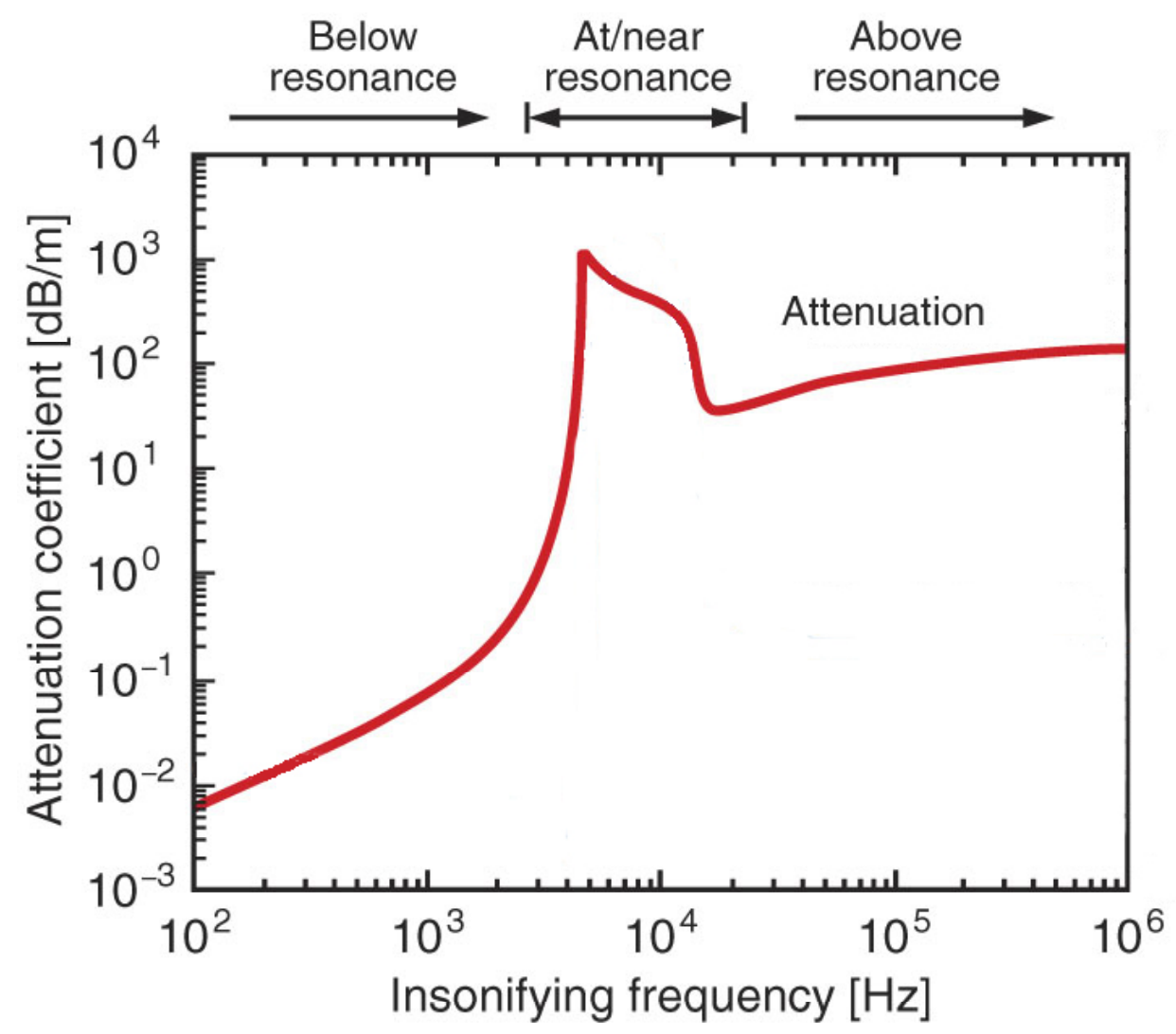

Figures 4 2: M odeled prediction of w ave attenuation coefficients in fine sand for a wide range of frequencies (m odified from Tuffin et al., 2000). $\mathrm{N}$ ote that the conditions $\mathrm{m}$ odeled here are not an exact analog for those in the collum ns. Rather the figure is included to illustrate the dram atic increase in attenuation possible when transm itting frequencies closely $\mathrm{m}$ atch the particular resonance frequency of bubbles in the sedim ent. 
As bubble growth continued, especially higher in the column with less overburden stress, the bubble radii increased to the point that the resonance frequencies were below those used here. In this case, attenuation due to scattering is no doubt the dominant contributor. At this point, however, the predicted attenuation due to scattering will be left to a later time and modeling exercise. Had a wider range of transducer frequencies been available, however, it is likely that losses due specifically to bubble damping could have been observed throughout the column. In fact, it seems likely that such a method (i.e. large bandwidth acoustic monitoring) would be the next logical step in monitoring and characterizing bubble formation and evolution. As bubble size dramatically influences the degree to which the subsurface is impacted, any ability to estimate bubble size would be of great use.

\subsection{Conclusions}

The above data and analysis illustrate that microbial activity can and does result in physical property changes that are detectable using geophysical means. In the case of this experiment, the geophysical technique used was that of monitoring transmitted acoustic wave amplitudes over the course of a microbial stimulation. The corroboration of various lines of evidence implies that significant gas generation is 
possible given appropriate conditions and that it is both measurable and quantifiable.

For now, it appears that this experiment has resulted in the very early stage development of a non-destructive and non-invasive means for characterizing stimulated microbial activity. With respect to microbial gas production, it appears that a unique and novel method now exists which may be used to assess the subsurface (or column-contained) impact of any produced gas. Furthermore, such a method may be readily incorporated into ongoing research activities designed to better understand gas-generating processes at both the laboratory and field scale.

In short, the first steps have been taken toward the development of a so-called Microbial Megascope. As such, we may now be in a better position to non-invasively examine the activity and impact of heretoforeunseen microbiological processes. 


\section{References}

Anderson, A.L., and L. D. Hampton, Acoustics of gas-bearing sediments, I. Background, J. Accoust. Soc. Am., 67(6), 1865-1889, 1980a.

Anderson, A. L., and L. D. Hampton, Acoustics of gas-bearing sediments, II. Measurements and models, J. Accoust. Soc. Am., 67(6), 1865-1889, 1980b.

Anderson, A.L, F. Abegg, J.A. Hawkins, and A.P. Lyons, Bubble populations and acoustic interaction with the gassy floor of Eckernförde Bay, Continental Shelf Research, 18(14-15), 1807-1838, 1998.

Anderson, J.N., and G.B. Wilson, The composition of dissolved gases in deep groundwaters and groundwater degassing, in "Saline Water and Gases in Crystalline Rocks", P. Fritz and S.K. Frape (Editors), Geological Association of Canada Special Paper 33, pp. 245-252, 1987.

Atkins, G.J., S.J. Prosser, and A. Barrie, Recent developments in the isotopic analysis of gases, Agron. Abstr., 84, 249-271, 1992. 
Baveye, P., P. Vandevivere, B.L. Hoyle, P.C. DeLeo, and D. Lozada, Environmental impact and mechanisms of the biological clogging of saturated soils and aquifer materials, Critical Reviews in Environmental Science and Technology, 28, 123-191, 1998.

Beckwith, C.W., and A.J. Baird, Effect of biogenic gas bubbles on water flow through poorly decomposed blanket peat, Water Resour. Res., 37, 551-558, 2001.

Blanchard, D.C., and L.D. Syzdek, Production of air bubbles of a specified size, Chem. Eng. Sci., 32, 1109-1112, 1977.

Chapelle, F.H., J.L., Zelibor Jr., D.J. Grimes, and L.L> Knobel, Bacteria in deep Coastal Plain sediments of Maryland: a possible source of $\mathrm{CO}_{2}$ to groundwater, Water Resour. Res., 23, 1625-1632, 1987.

Collins, R.E., Flow of fluid through porous materials, Petroleum Publishing company, Tulsa, Oklahoma, 1976.

Delozada, D. S., P. Vandevivere, P. Baveye, and S. Zinder, Decrease of the hydraulic conductivity of sand columns by Methanosarcina-Barkeri, World J. Microbiol. Biotechnol., 10, 325-333, 1994.

Doner, H.E., M.G. Volz, and A.D. McLaren, Column studies of denitrification in soil, Soil Biol. Biochem., 6, 341-346, 1974.

Doner, H.E., Disappearance of nitrate under transient conditions in columns of soil, Soil Biol. Biochem., 7, 257-259, 1975a. 
Doner, H.E., M.G. Volz, L.W. Belser, and J.P. Loken, Short term nitrate losses and associated microbial populations in soil columns, Soil Biol. Biochem., 7, 261-263, 1975b.

Doner, H.E., and A.D. McLaren, Temperature dependence of nitrate losses and denitrifier populations in soil columns, in "Environmental Biogeochemistry and Geomicrobiology, Volume 2: The Terrestrial Environment", W.E. Krumbein (Editor), Ann Arbor Science Publishers, Ann Arbor, MI, pp 573-582, 1978.

Dullien, F.A.L. Capillarity in porous media, Chapter 3, in "Porous Media: Fluid transport and pore structure", Academic Press, New York, New York, 1979.

Faybishenko, B.A., Hydraulic behavior of quasi-saturated soils in the presence of entrapped air: Laboratory experiments, Water Resour. Res., 31, 2421-2435, 1995.

Fry, V.A., J.S. Selker, and S.M. Gorelick, Experimental investigations for trapping oxygen gas in saturated porous media for in situ bioremediation, Water Resour. Res., 33, 2687-2696, 1997.

Gellar, J.T., Laboratory studies of groundwater degassing in replicas of natural fractured rock for linear flow geometry, LBNL Report 41386, E. O. Lawrence Berkeley National Laboratory, Berkeley, CA, 1998.

Johnston, D.H., and M.N. Toksöz, Definitions and terminology, in "Seismic Wave Attenuation, Chapter 1," D.H. Johnston and M.N. 
Toksöz (Editors), Society of Exploration Geophysicists, Tulsa, Oklahoma, pp. 1-5, 1981.

Jury, W.A., W.R. Gardner, and W.H. Gardner, Water flow in saturated soil, in "Soil Physics, Chapter 3," John Wiley and Sons, New York, New York, 1991.

Lyons, A.P., M.E. Duncan, and A.L. Anderson, Predictions of the acoustic scattering response of free-methane bubbles in muddy sediments, J. Accoust. Soc. Am., 99(1), 163-172, 1996.

Madigan, M.T., J.M., Martinko, and J. Parker, Biochemistry of dissimilative nitrate reduction, in "Brock Biology of Microorganisms, Chapter 15," D.M. Slyvia, J.J. Fuhrmann, P.G. Hartel, and D.A. Zuberer (Editors), Prentice Hall, Upper Saddle River, New Jersey, 1999.

Meyer, L., and S. Nakagawa, Mechanical and seismic properties of granular rock, LBNL Report 47642, E. O. Lawrence Berkeley National Laboratory, Berkeley, CA, 2000.

Myrold, D.D., Transformations of nitrogen, in "Principles and Applications of Soil Microbiology, Chapter 12," P.F. Corey (Editor), Prentice Hall, Upper Saddle River, New Jersey, 2000.

Nazaroff, W.W., and L. Alvarez-Cohen, Transformation processes, in "Environmental Engineering Science, Chapter 3," John Wiley and Sons, New York, New York, 2001. 
Reynolds, W.D., D.A. Brown, S.P. Mathur, and R.P. Overend, Effect of insitu gas accumulation on the hydraulic conductivity of peat, Soil Sci., 153, 397-408, 1992.

Richardson, M.D., and A.M. Davis, Modeling methane-rich sediments of Eckernförde Bay, Continental Shelf Research, 18(14-15), 1671-1688, 1998.

Rittmann, B.E., and P.L. McCart, Stoichiometry and bacterial energetics, in "Environmental biotechnology: principles and applications, Chapter 2," McGraw-Hill, New York, New York, pp. 126-135, 2001.

Ronen, D., B. Berkowitz, and M. Margaritz, The development and occurrence of gas bubbles in phreatic aquifers under natural flow conditions, Transport in Porous Media, 4, 295-306, 1989.

Seifert, P.K., J.T. Gellar, and L.R. Johnson, Effect of P-wave scattering on velocity and attenuation in unconsolidated sand saturated with immiscible liquids, Geophysics, 63, 161-170, 1998.

Smith, R.L., D.N. Miller, M.H. Brooks, M.A. Widdowson, and M.W. Killingstad, In situ stimulation of groundwater denitrification with formate to remediate nitrate contamination, Environ. Sci. Technol., 35, 196-203, 2001.

Tiedje, J.M., Denitrification, in "Methods of Soil Analysis, Part 2", A.L. Page (Editor), Agronomy Society of America, Madison, WI, pp 10111026, 1994. 
Trudell, M.R., R.W. Gillham, and J.A. Cherry, An in-situ study of the occurrence and rate of denitrification in a shallow unconfined sand aquifer, Journal of Hydrology, 83, 251-268, 1986.

Tuffin, M., A. Best, J. Dix, and J. Bull, Modeling temporal variability of $\mathrm{p}^{-}$ wave attenuation due to gas bubbles in a marine sediment, Proceedings of the $5^{\text {th }}$ European conference on underwater acoustics, Lyon, 2000.

Vogel, J.C., A.S. Talma, and T.H.E. Heaton, Gaseous nitrogen as evidence for denitrification in groundwater, Journal of Hydrology, 50, 191-200, 1981.

Wilkins, R.H. and M.D. Richardson, The influence of gas bubbles on sediment acoustic properties: in situ, laboratory, and theoretical results from Eckernförde Bay, Baltic sea, Continental Shelf Research, 18(14-15), 1859-1892, 1998.

Wilson, G.B., J.N. Andrews, and A.H. Bath, Dissolved gas evidence for denitrification in the Lincolnshire limestone groundwaters, eastern England, Journal of Hydrology, 113, 51-60, 1990.

Wilson, R.D., D.M. Mackay, and K.M. Scow, In situ biodegradation supported by diffusive oxygen release, Environ. Sci. Technol., 36, 190199, 2002. 


\section{Appendix A}

\section{Parameter and Data Tables}

The following tables contain information on the specific parameters used in the experiment. They include details of the sediment used, the defined growth medium used, and the column dimensions.

Table A.1: Sediment Parameters

\begin{tabular}{|cc|}
\hline Parameter & Measured Value \\
\hline \hline Composition & quartz $\left(\mathrm{SiO}_{2}\right)$ \\
Grain Size (diameter) & 150 to $250 \mu \mathrm{m}$ \\
Bulk Density & $1.8 \mathrm{gcm}^{-3}$ \\
Particle Density & $2.6 \mathrm{gcm}^{-3}$ \\
Porosity & $38 \%$ \\
\hline
\end{tabular}


Table A.2: Sediment Column Dimensions

\begin{tabular}{|cc|}
\hline Parameter (column type) & Measured Value \\
\hline \hline Composition (all) & Polycarbonate \\
Outer Diameter (all) & $6.35 \mathrm{~cm}$ \\
Inner Diameter (acoustic/control) & $6.03 \mathrm{~cm}$ \\
Inner Diameter (K/ $/$ Gas) & $5.08 \mathrm{~cm}$ \\
Total Column Length (all) & $30.48 \mathrm{~cm}$ \\
Sediment Height (acoustic/control) & $25.48 \mathrm{~cm}$ \\
Sediment Height (K $/$ Gas) & $30.48 \mathrm{~cm}$ \\
Pore Volume (acoustic/control) & $276.51 \mathrm{~cm}^{3}$ \\
Pore Volume (K/ $/$ Gas) & $234.76 \mathrm{~cm}^{3}$ \\
\hline
\end{tabular}

Table A.3: Composition of 100x Medium D Stock Solutions

40x Phosphate Buffer

\begin{tabular}{|cc|}
\hline Chemical & Concentration $(\mathrm{g} / \mathrm{L})$ \\
\hline \hline & 80 \\
potassium phosphate, monobasic & 140 \\
potassium phosphate, dibasic & \\
\hline
\end{tabular}


10x Mineral Salts

\begin{tabular}{|cc|}
\hline Chemical & Concentration $(\mathrm{g} / \mathrm{L})$ \\
\hline \hline ammonium sulfate & 10 \\
magnesium sulfate, heptahydrate & 5 \\
calcium nitrate, tetrahydrate & 0.354 \\
sodium nitrate & 20 \\
sodium acetate & 20 \\
\hline
\end{tabular}

1000x Trace Minerals

\begin{tabular}{|cc|}
\hline Chemical & Concentration $(\mathrm{g} / \mathrm{L})$ \\
\hline \hline manganese sulfate, monohydrate & 1 \\
boric acid & 0.062 \\
zinc sulfate, heptahydrate & 0.58 \\
copper(II) sulfate, pentahydrate & 0.25 \\
sodium molybdate, dihydrate & 0.24 \\
copper(II) nitrate, hexahydrate & 0.29 \\
nickel(II) sulfate, hexahydrate & 0.11 \\
sodium selenite & 0.035 \\
ferrous sulfate, heptahydrate & 1.36 \\
vanadium sulfate, dihydrate & 0.2 \\
hydrochloric acid & 1 drop $12 \mathrm{~N}$ \\
\hline
\end{tabular}


1000x Vitamins (OPCA)

\begin{tabular}{|cc|}
\hline Chemical & Concentration $(\mathrm{g} / \mathrm{L})$ \\
\hline \hline folic acid & 0.2 \\
pyridoxine hydrochloride & 1 \\
riboflavin & 0.5 \\
thiamine & 0.5 \\
nicotinic acid & 0.5 \\
pantothenic acid & 0.5 \\
vitamin B12 & 0.01 \\
p-aminobenzoic acid & 0.5 \\
thioctic acid & 0.5 \\
\hline
\end{tabular}

Table A.4: Proportions of Stock Solutions for 1L 1000x Medium D

100x Medium D $(1000 \mathrm{~mL})$

\begin{tabular}{|cc|}
\hline Stock Solution & Volume $(\mathrm{mL})$ \\
\hline \hline 40x Phosphate Buffer & 25 \\
10x Mineral Salts & 100 \\
1000x Trace Minerals & 1 \\
1000x Vitamins (OPCA) & 1 \\
De-lonized Water & 873 \\
\hline
\end{tabular}

